\title{
GEVREY SPACES AND THEIR INTERSECTIONS
}

\author{
A. F. M. TER ELST
}

(Received 11 February 1991)

Communicated by I. Raeburn

\begin{abstract}
For unbounded operators $A_{1}, \ldots, A_{d}$, Gevrey spaces $S_{\lambda_{1}, \ldots, \lambda_{d}}\left(A_{1}, \ldots, A_{d}\right)$ of order $\left(\lambda_{1}, \ldots, \lambda_{d}\right)$ are introduced, where the orders $\lambda_{1}, \ldots, \lambda_{d}$ need not be equal. These extend the notion of Gevrey space defined by Goodman and Wallach where $\lambda_{1}=\cdots=\lambda_{d}$. Several mild conditions on the operators $A_{1}, \ldots, A_{d}$ and the orders $\lambda_{1}, \ldots, \lambda_{d}$ are presented such that the equality $S_{\lambda_{1}, \ldots, \lambda_{d}}\left(A_{1}, \ldots, A_{d}\right)=\bigcap_{k=1}^{d} S_{\lambda_{k}}\left(A_{k}\right)$ is valid. Examples are included.

1991 Mathematics subject classification (Amer. Math. Soc.): 47 D 30, 22 E 45.
\end{abstract}

\section{Introduction}

In his well-known paper, [11], Nelson has introduced the concept of analytic vector relative to a finite set of operators as follows: Let $A_{1}, \ldots, A_{d}$ be (not necessarily bounded) operators in a normed space $E$. A vector $u \in E$ is called a $C^{\infty}$-vector for $\left\{A_{1}, \ldots, A_{d}\right\}$ if $u$ belongs to the domain $D\left(A_{i_{1}}\right.$ 。 $\left.\cdots \circ A_{i_{n}}\right)$ of the operator $A_{i_{1}} \circ \cdots \circ A_{i_{n}}$ for all $n \in \mathbb{N}_{0}$ and all $i_{1}, \ldots, i_{n} \in$ $\{1, \ldots, d\}$. Let $D^{\infty}\left(A_{1}, \ldots, A_{d}\right)$ denote the space of all $C^{\infty}$-vectors for $\left\{A_{1}, \ldots, A_{d}\right\}$. A vector $u \in E$ is an analytic vector relative to $\left\{A_{1}, \ldots, A_{d}\right\}$ if $u$ is a $C^{\infty}$-vector and there exist constants $c, t>0$ such that for all $n \in \mathbb{N}_{0}$ and all $i_{1}, \ldots, i_{n} \in\{1, \ldots, d\}$ we have

$$
\left\|A_{i_{1}} \circ \cdots \circ A_{i_{n}} u\right\| \leq c t^{n} n ! \text {. }
$$

An extension of the concept of analytic vector, given by Goodman and Wallach [10], is derived by replacing $n$ ! in the above expression by $n !^{\lambda}$. (C) 1993 Australian Mathematical Society 0263-6115/93 \$A2.00+0.00 
Thus we arrive at the concept of Gevrey vector of order $\lambda \geq 0$ relative to $\left\{A_{1}, \ldots, A_{d}\right\}$. In the latter definition of Gevrey vector the operators are treated on the same level.

The notion of Gevrey vector is based on the notion of Gevrey function whose definition according to Gevrey [6], is the following. Let $\lambda_{1}, \ldots, \lambda_{d} \geq$ 0 . A function $f$ defined on an open subset $U$ of $\mathbb{R}^{d}$ is called a Gevrey function of order $\left(\lambda_{1}, \ldots, \lambda_{d}\right)$ (in [6], a function of class $\lambda_{1}$ in $x_{1}, \ldots$, of class $\lambda_{d}$ in $x_{d}$ ) if $f$ is infinitely differentiable and for every compact subset $K$ of $U$ there exist constants $c, t>0$ such that for all $n_{1}, \ldots, n_{d} \in \mathbb{N}_{0}$ and all $x \in K$ we have

$$
\left|\left(D_{1}^{n_{1}} \ldots D_{d}^{n_{d}} f\right)(x)\right| \leq c t^{n_{1}+\cdots+n_{d}} n_{1} !^{\lambda_{1}} \ldots n_{d} !^{\lambda_{d}} .
$$

Here $D_{k}$ denotes partial differentiation with respect to the $k$-th coordinate. We see that in Gevrey's original definition the variables $\left(x_{1}, \ldots, x_{d}\right)$ are not treated on the same level and thus the following natural generalization of the concept of Gevrey vector arises. Let $A_{1}, \ldots, A_{d}$ be (possibly not everywhere defined) operators in locally convex topological vector space $E$. Let $\lambda_{1}, \ldots, \lambda_{d} \geq 0$. A vector $u \in E$ is called a Gevrey vector of order $\left(\lambda_{1}, \ldots, \lambda_{d}\right)$ relative to $\left(A_{1}, \ldots, A_{d}\right)$ if for every continuous seminorm $p$ on $E$ there exist constants $c, t>0$ such that for all $n \in \mathbb{N}_{0}$ and all $i_{1}, \ldots, i_{n} \in\{1, \ldots, d\}$ the vector $u$ belongs to the domain of the operator $A_{i_{1}} \circ \cdots \circ A_{i_{n}}$ and $p\left(A_{i_{1}} \circ \cdots \circ A_{i_{n}} u\right) \leq c t^{n} n_{1} !^{\lambda_{1}} \cdots n_{d} !^{\lambda_{d}}$, where $n_{k}:=\operatorname{card}\{l \in$ $\{1, \ldots, n\}$ : $\left.i_{l}=k\right\}$ for all $k \in\{1, \ldots, d\}$. By $S_{\lambda_{1}, \ldots, \lambda_{d}}\left(A_{1}, \ldots, A_{d}\right)$ we denote the space of all Gevrey vectors of order $\left(\lambda_{1}, \ldots, \lambda_{d}\right)$ relative to $\left(A_{1}, \ldots, A_{d}\right)$.

Trivially, a Gevrey vector of order $\left(\lambda_{1}, \ldots, \lambda_{d}\right)$ relative to $\left(A_{1}, \ldots, A_{d}\right)$ is a Gevrey vector of order $\lambda_{k}$ relative to $A_{k}$ for all $k$, so $S_{\lambda_{1}, \ldots, \lambda_{d}}\left(A_{1}, \ldots\right.$, $\left.A_{d}\right) \subset \bigcap_{k=1}^{d} S_{\lambda_{k}}\left(A_{k}\right)$. So the following interesting problem comes up: find appropriate conditions on $\lambda_{1}, \ldots, \lambda_{d}$ and $A_{1}, \ldots, A_{d}$ such that

$$
S_{\lambda_{1}, \ldots, \lambda_{d}}\left(A_{1}, \ldots, A_{d}\right)=\bigcap_{k=1}^{d} S_{\lambda_{k}}\left(A_{k}\right) .
$$

In Sections 3 and 4 such conditions are presented for skew-hermitian operators. Our strategy is as follows. First mild conditions are presented such that

$$
S_{\lambda_{1}, \ldots, \lambda_{d}}\left(A_{1}, \ldots, A_{d}\right)=S_{\lambda_{1}, \ldots, \lambda_{n}}\left(A_{1}, \ldots, A_{n}\right) \cap S_{\lambda_{n+1}, \ldots, \lambda_{d}}\left(A_{n+1}, \ldots, A_{d}\right)
$$

for some $n \in\{1, \ldots, d-1\}$. Then from this we derive stronger conditions such that equality (1) holds. In the literature these problems have been 
looked at only in case $\lambda_{1}=\cdots=\lambda_{d}=1$ by [7] and [4] in order to prove separate and joint analyticity for representations of Lie groups in certain cases. In those papers, the operators $A_{k}$ are infinitesimal generators for the representation. (Cf. Theorem 24.) However, in general equality (1) is not valid. (See Example 7.)

In general, the $C^{\infty}$-domain $D^{\infty}\left(A_{1}, \ldots, A_{d}\right)$ is trivial, i.e. it contains only the zero vector, hence the Gevrey space $S_{\lambda_{1}, \ldots, \lambda_{d}}\left(A_{1}, \ldots, A_{d}\right)$ is also trivial. In Section 5 we present several examples of non-trivial Gevrey spaces by taking for $A_{1}, \ldots, A_{d}$ infinitesimal generators of a representation of a Lie group. In Section 6 we consider the $a x+b$ group. Another interesting illustration is the Heisenberg group. This group has served as a major source of inspiration and is used frequently in the present paper.

\section{Multi-indices and Gevrey spaces}

Let $d \in \mathbb{N}$ and let $A_{1}, \ldots, A_{d}$ be (possibly not everywhere defined) operators in a locally convex topological vector space $E$. Since we do not assume that the operators $A_{1}, \ldots, A_{d}$ commute, we have to take care of the order in which the operators occur. To this end we introduce the concept of multi-index.

Let $V$ be a non-empty finite set. We define the set $M(V)$ of multi-indices over $V$ by $M(V):=\bigcup_{n \in \mathrm{N}_{0}} V^{n}$. Here $V^{0}$ denotes the set with one element, called the empty sequence, which is denoted by ( ). For $\alpha=\left(j_{1}, \ldots, j_{n}\right) \in$ $M(V)$ define the length $\|\alpha\|$ of $\alpha$ by $\|\alpha\|:=n$ and for $v \in V$ define the $v$-length $\|\alpha\|_{v}$ of $\alpha$ by $\left\|\left(j_{1}, \ldots, j_{n}\right)\right\|_{v}:=\operatorname{card}\left\{i \in\{1, \ldots, n\}: j_{i}=v\right\}$. For $v \in V$ let $\lambda_{v} \geq 0$. For $\alpha \in M(V)$ define

$$
\alpha !^{\lambda}:=\prod_{v \in V}\|\alpha\|_{v} !^{\lambda_{v}}
$$

In a natural way we define an operation on $M(V)$ : for $\alpha, \beta \in M(V)$ define the concatenation $\langle\alpha, \beta\rangle$ of $\alpha$ and $\beta$ by

$$
\left\langle\left(j_{1}, \ldots, j_{n}\right),\left(k_{1}, \ldots, k_{m}\right)\right\rangle:=\left(j_{1}, \ldots, j_{n}, k_{1}, \ldots, k_{m}\right)
$$

for all $n, m \in \mathbb{N}_{0}$ and all $j_{1}, \ldots, j_{n}, k_{1}, \ldots, k_{m} \in V$. So $M(V)$ is a monoid with identity ( ). Define similarly the concatenation $\left\langle\alpha_{1}, \ldots, \alpha_{p}\right\rangle$ of $\alpha_{1}, \ldots, \alpha_{p} \in M(V)$.

Finally, for $\alpha \in M(V)$ define the reverse $\alpha^{\mathrm{r}}$ of $\alpha$ by $\left(j_{1}, \ldots, j_{n}\right)^{\mathrm{r}}:=$ $\left(j_{n}, \ldots, j_{1}\right)$. In Section 4 we introduce some more operations on $M(V)$.

Now let $V:=\{1, \ldots, d\}$. For $\alpha \in M(V)$ define the operator $A_{\alpha}$ by 
$A_{(\mathrm{)})}:=I$ and

$$
A_{\left(j_{1}, \ldots, j_{n}\right)}:=A_{j_{1}} \circ \cdots \circ A_{j_{n}} \quad\left(n \in \mathbb{N}, j_{1}, \ldots, j_{n} \in V\right) .
$$

Then the joint $C^{\infty}$-domain of the operators $A_{1}, \ldots, A_{d}$ equals

$$
D^{\infty}\left(A_{1}, \ldots, A_{d}\right):=\bigcap_{\alpha \in M(V)} D\left(A_{\alpha}\right) .
$$

Now we define our Gevrey spaces. Let $\lambda_{1}, \ldots, \lambda_{d} \geq 0$. A vector $u \in E$ is called a Gevrey vector of order $\left(\lambda_{1}, \ldots, \lambda_{d}\right)$ relative to $\left(A_{1}, \ldots, A_{d}\right)$ if $u \in D^{\infty}\left(A_{1}, \ldots, A_{d}\right)$ and for every continuous seminorm $p$ on $E$ there exist constants $c, t>0$ such that for all $\alpha \in M(V)$ we have

$$
p\left(A_{\alpha} u\right) \leq c t^{\|\alpha\|} \alpha !^{\lambda} \text {. }
$$

The space of all Gevrey vectors of order $\left(\lambda_{1}, \ldots, \lambda_{d}\right)$ relative to $\left(A_{1}, \ldots\right.$, $\left.A_{d}\right)$ is denoted by $S_{\lambda_{1}, \ldots, \lambda_{d}}\left(A_{1}, \ldots, A_{d}\right)$. Note that if $E$ is a normed space, then

$$
\begin{aligned}
& S_{\lambda_{1}, \ldots, \lambda_{d}}\left(A_{1}, \ldots, A_{d}\right) \\
& \quad=\left\{u \in D^{\infty}\left(A_{1}, \ldots, A_{d}\right): \exists_{c, i>0} \forall_{\alpha \in M(V)}\left[\left\|A_{\alpha} u\right\| \leq c t^{\|\alpha\|} \alpha !^{\lambda}\right]\right\} .
\end{aligned}
$$

In case the operators $A_{1}, \ldots, A_{d}$ are skew-Hermitian operators in a Hilbert space, we have the following important characterization of $S_{\lambda_{1}, \ldots, \lambda_{d}}\left(A_{1}, \ldots, A_{d}\right)$.

Lemma 1. Let $A_{1}, \ldots, A_{d}$ be skew-Hermitian operators in a Hilbert space $H$. Let $\lambda_{1}, \ldots, \lambda_{d} \geq 0$. Let $u \in H$. Then $u \in S_{\lambda_{1}, \ldots, \lambda_{d}}\left(A_{1}, \ldots, A_{d}\right)$ if and only if $u \in D^{\infty}\left(A_{1}, \ldots, A_{d}\right)$ and there exist $c, t>0$ such that for all $\alpha \in M(\{1, \ldots, d\})$ we have $\left|\left(A_{\alpha} u, u\right)\right| \leq c t^{\|\alpha\|} \alpha !^{\lambda}$.

Proof. We only prove the "if" part. Let $\alpha \in M(\{1, \ldots, d\})$. Then

$$
\begin{aligned}
\left\|A_{\alpha} u\right\|^{2} & =\left|\left(A_{\left\langle\alpha^{r}, \alpha\right\rangle} u, u\right)\right| \leq c t^{2\|\alpha\|}\left(2\|\alpha\|_{1}\right) !^{\lambda_{1}} \cdots\left(2\|\alpha\|_{d}\right) !^{\lambda_{d}} \\
& \left.\leq\left[\sqrt{c}\left(2^{\lambda_{1}+\cdots+\lambda_{d}} t\right)\right)^{\|\alpha\|} \alpha !^{\lambda}\right]^{2} .
\end{aligned}
$$

We finish this section with some examples.

EXAMPLE 2. Let $U$ be an open subset of $\mathbb{R}^{d}$. Let $C(U)$ be the locally convex topological vector space of all continuous functions on $U$ with the topology of uniform convergence on compacta. Let $D_{1}, \ldots, D_{d}$ be the partial differentiation operators in $C(U)$ with domain $C^{1}(U)$. Then $S_{\lambda_{1}, \ldots, \lambda_{d}}\left(D_{1}, \ldots, D_{d}\right)$ is the space of Gevrey functions of order $\left(\lambda_{1}, \ldots, \lambda_{d}\right)$. (See [6].) 
EXAMPLe 3. Let $A$ be an operator in a Hilbert space $H$. Then $S_{0}(A)$ is the set of all bounded vectors for $A$. (See [3, Section 26].)

EXAMPLe 4. Let $A_{1}, \ldots, A_{d}$ be $d$ operators in a Hilbert space $H$ and let $\lambda \geq 0$. Then $S_{\lambda, \ldots, \lambda}\left(A_{1}, \ldots, A_{d}\right)$ is equal to the set of Gevrey vectors of order $\lambda$ relative to $\left\{A_{1}, \ldots, A_{d}\right\}$. (See [10, Section 1].) In particular, for $\lambda=1$, the set $S_{1, \ldots, 1}\left(A_{1}, \ldots, A_{d}\right)$ is equal to the set of analytic vectors for $\left\{A_{1}, \ldots, A_{d}\right\}$. (See $[11$, Section 2$]$.)

EXAMPLe 5. Let $(X, \mathscr{B}, m)$ be a measure space and let $h$ be a measurable function. Let $H:=L^{2}(X, m)$ and let $A$ be the multiplication operator by $h$. Let $\lambda>0$. Then $S_{\lambda}(A)=\left\{e^{-t|h|^{1 / \lambda}} f: f \in L^{2}(X, m)\right\}$.

EXAMPLE 6. Let $Q$ be the multiplication operator by the function $x \mapsto x$ in $L^{2}(\mathbb{R})$. Let $D$ be the skew-adjoint differentiation operator in $L^{2}(\mathbb{R})$. Then $D^{\infty}(Q, D)$ is equal to $S$ chwartz' space $S(\mathbb{R})$, that is, the space of all infinitely differentiable functions $\varphi$ defined on $\mathbb{R}$ such that $\sup \left\{\left|x^{k} \varphi^{(l)}(x)\right|\right.$ : $x \in \mathbf{R}\}<\infty$ for all $k, l \in \mathbb{N}_{0}$. (See [7, p. 65].) Let $\alpha, \beta \geq 0$ and suppose that $\alpha+\beta \geq 1$. By [2, Theorem 4.3] we obtain that

$$
S_{\alpha, \beta}(Q, D)=\left\{\varphi \in D^{\infty}(Q, D):=\exists_{c, t>0} \forall_{k, l \in \mathrm{N}_{0}}\left[\left\|Q^{k} D^{l} \varphi\right\| \leq c t^{k+l} k !^{\alpha} l !^{\beta}\right]\right\} \text {. }
$$

(See also the proof of Theorem 11.) So the Gevrey space $S_{\alpha, \beta}(Q, D)$ is equal to the Gelfand-Shilov space $S_{\alpha}^{\beta}$, which is defined by

$$
S_{\alpha}^{\beta}:=\left\{\varphi \in S(\mathbb{R}): \exists_{n \in \mathbf{N}} \sup _{\substack{k, l \in \mathbf{N}_{0} \\ x \in \mathbb{R}}}\left|x^{k} \varphi^{(l)}(x)\right| n^{-k-l} k^{-k \alpha} l^{-l \beta}<\infty\right\} .
$$

(See [5, Section IV.3.3] and [16, Section 29.5].)

EXAMPLE 7. Let $H:=l^{2}(\mathbb{N})$ with standard basis $e_{1}, e_{2}, \ldots$ Let $A$ be the continuous shift operator, determined by $A e_{n}:=e_{n+1}$ for all $n \in \mathbb{N}$ and let $B$ be the self-adjoint multiplication operator determined by $B e_{n}:=n !^{2} e_{n}$ for all $n \in \mathbb{N}$. Then $e_{1} \in S_{1}(A) \cap S_{1}(B)$. But $e_{1} \notin S_{1,1}(A, B)$ since $\left\|B A^{n-1} e_{1}\right\|=n !^{2}$ for all $n \in \mathbb{N}$.

\section{Gevrey spaces relative to coupled sets of skew-Hermitian operators}

As said in the introduction, the main step in the proof of equality (1) is equality (2). The following theorem is the first of such intersection theorems.

Theorem 8. Let $d_{1}, d_{2} \in \mathbb{N}$ and let $X_{1}, \ldots, X_{d_{1}}, Y_{1}, \ldots, Y_{d_{2}}$ be skewhermitian operators in a Hilbert space defined on a common invariant domain. 
Suppose

$$
\left[X_{i}, Y_{j}\right] \in \operatorname{span}\left(\left\{X_{1}, \ldots, X_{d_{1}}, Y_{1}, \ldots, Y_{d_{2}}\right\}\right)
$$

for all $i \in\left\{1, \ldots, d_{1}\right\}$ and $j \in\left\{1, \ldots, d_{2}\right\}$. Let $\lambda, \mu \geq 1$. Then

$$
\begin{aligned}
& S_{\lambda, \ldots, \lambda, \mu, \ldots, \mu}\left(X_{1}, \ldots, X_{d_{1}}, Y_{1}, \ldots, Y_{d_{2}}\right) \\
& \quad=S_{\lambda, \ldots, \lambda}\left(X_{1}, \ldots, X_{d_{1}}\right) \cap S_{\mu, \ldots, \mu}\left(Y_{1}, \ldots, Y_{d_{2}}\right) .
\end{aligned}
$$

This theorem yields three extensions of a result, [4, Theorem 2], of Flato and Simon at once. Indeed, they proved the above intersection theorem in the following very special case: $\lambda=\mu=1 ; \mathfrak{g}:=\operatorname{span}\left(\left\{X_{1}, \ldots, X_{d_{1}}, Y_{1}, \ldots\right.\right.$, $\left.\left.Y_{d_{2}}\right\}\right)$ is an integrable Lie algebra and both span $\left(\left\{X_{1}, \ldots, X_{d_{1}}\right\}\right)$ and span $\left(\left\{Y_{1}, \ldots, Y_{d_{2}}\right\}\right)$ are subalgebras of $\mathfrak{g}$. However, the theorem of Flato and Simon is also valid for representations in a Banach space. The essence of the proof in [4] is that for each element of $S_{1, \ldots, 1}\left(X_{1}, \ldots, X_{d_{1}}\right) \cap S_{1, \ldots, 1}\left(Y_{1}, \ldots\right.$, $Y_{d_{2}}$ ) a function is constructed, which is separately real analytic in a uniform sense and because of a result of Browder ([1]) this function can be shown to be jointly real analytic. The proof of our more general intersection theorem is based on totally different techniques.

Proof of Theorem 8. Let $V_{1}:=\left\{1, \ldots, d_{1}\right\}, V_{2}:=\left\{1, \ldots, d_{2}\right\}$ and $V:=\{1, \ldots, d\}$, where $d:=d_{1}+d_{2}$. Let $Z_{1}:=X_{1}, \ldots, Z_{d_{1}}:=X_{d_{1}}$, $Z_{d_{1}+1}:=Y_{1}, \ldots, Z_{d}:=Y_{d_{2}}$. For all $k, m \in \mathbb{N}_{0}$ we define the subset $U_{k, m}$ of $M(V)$ by

$$
U_{k, m}:=\left\{\gamma \in M(V): \sum_{i=1}^{d_{1}}\|\gamma\|_{i}=k \text { and } \sum_{i=d_{1}+1}^{d}\|\gamma\|_{i}=m\right\} .
$$

By assumption, for all $i \in V_{1}$ and $j \in V_{2}$ there exist $c_{i, j}^{1}, \ldots, c_{i, j}^{d_{1}}, b_{i, j}^{1}, \ldots$, $b_{i, j}^{d_{2}} \in \mathbb{R}$ such that

$$
\left[X_{i}, Y_{j}\right]=\sum_{l=1}^{d_{1}} c_{i, j}^{l} X_{l}+\sum_{l=1}^{d_{2}} b_{i, j}^{l} Y_{l} .
$$

Let $M:=1+\max \left\{\left|c_{i, j}^{l}\right|: i, l \in V_{1}, j \in V_{2}\right\}+\max \left\{\left|b_{i, j}^{l}\right|: i \in V_{1}, j, l \in V_{2}\right\}$.

Let $u \in S_{\lambda, \ldots, \lambda}\left(X_{1}, \ldots, X_{d_{1}}\right) \cap S_{\mu, \ldots, \mu}\left(Y_{1}, \ldots, Y_{d_{2}}\right)$. Then there exist $c, t>0$ such that $\left\|X_{\alpha} u\right\| \leq c t^{\|\alpha\|}\|\alpha\| !^{\lambda}$ and $\left\|Y_{\beta} u\right\| \leq c t^{\|\beta\|}\|\beta\| !^{\mu}$ for all $\alpha \in M\left(V_{1}\right)$ and $\beta \in M\left(V_{2}\right)$. We may assume that $t \geq M d$. For $N \in \mathbb{N}_{0}$ let hypothesis $P(N)$ state:

$$
\begin{gathered}
\left|\left(Y_{\beta} Z_{\gamma} X_{\alpha} u, u\right)\right| \leq c^{2} 3^{\|\gamma\|} t^{\|\alpha\|+\|\beta\|+\|\gamma\|}(\|\alpha\|+k) !^{\lambda}(\|\beta\|+m) !^{\mu} \\
\text { for all } k, m \in \mathbb{N}_{0}, \alpha \in M\left(V_{1}\right), \quad \beta \in M\left(V_{2}\right) \text { and } \gamma \in U_{k, m} \\
\text { such that } k+m=N .
\end{gathered}
$$


For $\alpha \in M\left(V_{1}\right)$ and $\beta \in M\left(V_{2}\right)$ we obtain by Schwarz' inequality

$$
\left|\left(Y_{\beta} Z_{(} X_{\alpha} u, u\right)\right|=\left|\left(X_{\alpha} u, Y_{\beta^{r}} u\right)\right| \leq\left\|X_{\alpha} u\right\|\left\|Y_{\beta^{r}} u\right\| \leq c t^{\|\alpha\|}\|\alpha\| !^{\lambda} c t^{\|\beta\|}\|\beta\| !^{\mu} .
$$

So hypothesis $P(0)$ holds, because $U_{0,0}=\{()\}$.

Let $N \in \mathbb{N}$ and suppose hypothesis $P(N-1)$ holds. Let $k, m \in \mathbb{N}_{0}$, $\alpha \in M\left(V_{1}\right), \beta \in M\left(V_{2}\right), \gamma \in U_{k, m}$ and suppose $k+m=N$. If $k=0$ or $m=0$, inequality (3) follows by hypothesis $P(0)$. So we may assume that $k \neq 0$ and $m \neq 0$.

Suppose $k \geq m$. By pushing the rightmost operator $X_{x}$ in $Z_{y}$ to the right and taking in account all possible commutators, we see that there exist $x \in$ $V_{1}, \delta \in U_{k-1, m}, c_{1}, \ldots, c_{d_{1} m} \in \mathbb{C}, \theta_{1}, \ldots, \theta_{d_{1} m} \in U_{k, m-1}, b_{1}, \ldots, b_{d_{2} m} \in$ $\mathbb{C}$ and $\eta_{1}, \ldots, \eta_{d_{2} m} \in U_{k-1, m}$ such that

$$
Z_{\gamma}=Z_{\delta} X_{x}+\sum_{p=1}^{d_{1} m} c_{p} Z_{\theta_{p}}+\sum_{q=1}^{d_{2} m} b_{q} Z_{\eta_{q}}
$$

and $\left|c_{p}\right| \leq M$ and $\left|b_{q}\right| \leq M$ for all $p, q$. Now by induction hypothesis $P(N-1)$ and the inequality $d M \leq t$ we obtain that:

$$
\begin{aligned}
\left|\left(Y_{\beta} Z_{\gamma} X_{\alpha} u, u\right)\right| & \\
\leq & \left|\left(Y_{\beta} Z_{\delta} X_{\langle x, \alpha)} u, u\right)\right|+\sum_{p=1}^{d_{1} m}\left|c_{p}\right|\left|\left(Y_{\beta} Z_{\theta_{p}} X_{\alpha} u, u\right)\right|+\sum_{q=1}^{d_{2} m}\left|b_{q}\right|\left|\left(Y_{\beta} Z_{\eta_{q}} X_{\alpha} u, u\right)\right| \\
\leq & c^{2} 3^{\|\gamma\|-1} t^{\|\alpha\|+\|\beta\|+\|\gamma\|}(\|\alpha\|+k) !^{\lambda}(\|\beta\|+m) !^{\mu} \\
& +d_{1} m M c^{2} 3^{\|\gamma\|-1} t^{\|\alpha\|+\|\beta\|+\|\gamma\|-1}(\|\alpha\|+k) !^{\lambda}(\|\beta\|+m-1) !^{\mu} \\
(5) \quad & +d_{2} m M c^{2} 3^{\|\gamma\|-1} t^{\|\alpha\|+\|\beta\|+\|\gamma\|-1}(\|\alpha\|+k-1) !^{\lambda}(\|\beta\|+m) !^{\mu} \\
\leq & c^{2} 3^{\|\gamma\|-1} t^{\|\alpha\|+\|\beta\|+\|\gamma\|}(\|\alpha\|+k) !^{\lambda}(\|\beta\|+m) !^{\mu} \cdot \\
& \cdot\left(1+\frac{m}{(\|\beta\|+m)^{\mu}}+\frac{m}{(\|\alpha\|+k)} \frac{(\|\alpha\|+k)}{(\|\alpha\|+k)^{\lambda}}\right) \\
\leq & c^{2} 3^{\|\gamma\|} t^{\|\alpha\|+\|\beta\|+\|\gamma\|}(\|\alpha\|+k) !^{\lambda}(\|\beta\|+m) !^{\mu} .
\end{aligned}
$$

In case $k \leq m$ a similar argument can be used by decomposing $Z_{\gamma}=$ $Y_{y} Z_{\delta}+$ "small terms". This proves hypothesis $P(N)$.

In particular, for all $k, m \in \mathbb{N}_{0}$ and $\gamma \in U_{k, m}$ we obtain that $\left|\left(Z_{\gamma} u, u\right)\right| \leq$ $c^{2}(3 t)^{\|v\|} k !^{\lambda} m !^{\mu}$. Now by Lemma 1 we derive that $u \in S_{\lambda, \ldots, \lambda, \mu, \ldots, \mu}\left(Z_{1}, \ldots\right.$, $Z_{d}$.

COROLlaRY 9. Let $\mathfrak{g}$ be a solvable real Lie algebra of skew-Hermitian operators defined on a common invariant domain. Let $X_{1}, \ldots, X_{d}$ be a basis in $\mathfrak{g}$ 
such that $\mathscr{L}_{i}:=\operatorname{span}\left(\left\{X_{1}, \ldots, X_{i}\right\}\right)$ is a subalgebra of $\mathfrak{g}$ and $\mathscr{L}_{i}$ is an ideal in $\mathscr{L}_{i+1}$ for all $i \in\{1, \ldots, d\}$. Let $\lambda \geq 1$. Then $S_{\lambda, \ldots, \lambda}\left(X_{1}, \ldots, X_{d}\right)=$ $\bigcap_{k=1}^{d} S_{\lambda}\left(X_{k}\right)$.

We apply the arguments of the proof of the above theorem for two operators which satisfy the Heisenberg commutation relations.

Lemma 10. Let $X, Y$ be skew-Hermitian operators in a Hilbert space defined on a common invariant domain. Suppose $[X, Y]=i I$. Let $\lambda, \mu \geq 0$ and suppose that $\lambda+\mu \geq 1$. Then $S_{\lambda, \mu}(X, Y)=S_{\lambda}(X) \cap S_{\mu}(Y)$.

Proof. The proof of this theorem is similar to the proof of the previous theorem, except that now we can take $\theta_{p} \in U_{k-1, m-1}$ and $b_{q}=0$ for all $p, q$ in the equality (4). Hence in inequality (5) we now obtain the factor

$$
\left(1+\frac{m}{m^{\lambda+\mu}} \frac{k^{\lambda}}{(\|\alpha\|+k)^{\lambda}} \frac{m^{\mu}}{(\|\beta\|+m)^{\mu}} \frac{m^{\lambda}}{k^{\lambda}}\right)
$$

which is less than or equal to 2 .

Now we obtain easily the following theorem, which has been proved firstly by Van Eijndhoven, ([2, Theorem 4.5]), in case $\alpha>0$ and $\beta>0$.

Theorem 11. Let $Q, D$ be the operators in $L^{2}(\mathbb{R})$ as in Example 6. Let $\alpha, \beta \geq 0$ and suppose $\alpha+\beta \geq 1$. Then $S_{\alpha}^{\beta}=S_{\alpha, \beta}(Q, D)=S_{\alpha}(Q) \cap S_{\beta}(D)$.

Proof. Let $X$ and $Y$ be the restrictions of the operators $-i Q$ and $D$ to Schwartz' space $S(\mathbb{R})$ respectively. Then $S_{\alpha, \beta}(X, Y)=S_{\alpha}(X) \cap S_{\beta}(Y)$ by Lemma 10 . Since $D^{\infty}(X, Y)=D^{\infty}(Q, D)=S(\mathbb{R})=D^{\infty}$.(Q) $\cap D^{\infty}(D)$, by [7, Section 6], we obtain that $S_{\alpha, \beta}(Q, D)=S_{\alpha}(Q) \cap S_{\beta}(D)$. So $S_{\alpha}^{\beta} \subset$ $S_{\alpha}(Q) \cap S_{\beta}(D)=S_{\alpha, \beta}(Q, D) \subset S_{\alpha}^{\beta}$, and the theorem is proved.

The following theorem can be proved in the same spirit as Theorem 8 .

THeOREM 12. Let $X_{1}, \ldots, X_{d_{1}}, Y_{1}, \ldots, Y_{d_{2}}$ be operators in a Hilbert space defined on a common invariant domain. Suppose all operators $X_{1}, \ldots$, $X_{d_{1}}, Y_{1}, \ldots, Y_{d_{2}}$ are Hermitian or skew-Hermitian and suppose $\left[X_{i}, Y_{j}\right]=0$ for all $i \in\left\{1, \ldots, d_{1}\right\}$ and $j \in\left\{1, \ldots, d_{2}\right\}$. Let $\lambda_{1}, \ldots, \lambda_{d_{1}}, \mu_{1}, \ldots, \mu_{d_{2}}$ $\geq 0$. Then $S_{\lambda_{1}, \ldots, \lambda_{d_{1}}, \mu_{1}, \ldots, \mu_{d_{2}}}\left(X_{1}, \ldots, X_{d_{1}}, Y_{1}, \ldots, Y_{d_{2}}\right)=S_{\lambda_{1}, \ldots, \lambda_{d_{1}}}\left(X_{1}, \ldots\right.$, $\left.X_{d_{1}}\right) \cap S_{\mu_{1}, \ldots, \mu_{d_{2}}}\left(Y_{1}, \ldots, Y_{d_{2}}\right)$. 
Proof. Now we can take the constants $c_{p}=b_{q}=0$ in equality (4) and so the factor in inequality (5) becomes 1 .

Using Lemma 10 and Theorem 12 the following version of Theorem 11 for Gelfand-Shilov spaces in several variables can be proved: let $d \in \mathbb{N}$, for $k \in\{1, \ldots, d\}$ let $Q_{k}$ be the multiplication operator in $L^{2}\left(\mathbb{R}^{d}\right)$ by the function $x \mapsto x_{k}$ and let $P_{k}$ be the Fourier transform of $Q_{k}$, that is, $P_{k}:=\mathbb{F}^{-1} Q_{k} \mathbb{F}$. Let $\alpha_{1}, \ldots, \alpha_{d}, \beta_{1}, \ldots, \beta_{d} \geq 0$ and suppose $\alpha_{k}+\beta_{k} \geq 1$ for all $k$. Then

$$
\begin{aligned}
S_{\alpha_{1}, \ldots, \alpha_{d}}^{\beta_{1}, \ldots, \beta_{d}} & =S_{\alpha_{1}, \ldots, \alpha_{d}, \beta_{1}, \ldots, \beta_{d}}\left(Q_{1}, \ldots, Q_{d}, P_{1}, \ldots, P_{d}\right) \\
& =\bigcap_{k=1}^{d} S_{\alpha_{k}}\left(Q_{k}\right) \cap \bigcap_{k=1}^{d} S_{\beta_{k}}\left(P_{k}\right) .
\end{aligned}
$$

Corollary 13. Let $X_{1}, \ldots, X_{d}$ be commuting operators in a Hilbert space defined on a common invariant domain. Suppose all operators $X_{1}, \ldots, X_{d}$ are Hermitian or skew-Hermitian. Let $\lambda_{1}, \ldots, \lambda_{d} \geq 0$. Then $S_{\lambda_{1}, \ldots, \lambda_{d}}\left(X_{1}, \ldots, X_{d}\right)=\bigcap_{k=1}^{d} S_{\lambda_{k}}\left(X_{k}\right)$.

Corollary 14. Let $A_{1}, \ldots, A_{d}$ be strongly commuting self-adjoint operators in a Hilbert space, that is, self-adjoint operators whose spectral projections commute, or equivalently, self-adjoint operators whose Cayley transforms commute. Let $\lambda_{1}, \ldots, \lambda_{d} \geq 0$. Then

$$
S_{\lambda_{1}, \ldots, \lambda_{d}}\left(A_{1}, \ldots, A_{d}\right)=\bigcap_{k=1}^{d} S_{\lambda_{k}}\left(A_{k}\right) \text {. }
$$

Proof. For $k \in\{1, \ldots, d\}$ let $X_{k}$ be the restriction of $A_{k}$ to $D^{\infty}\left(A_{1}\right.$, $\left.\ldots, A_{d}\right)$. By Corollary 13 we obtain $S_{\lambda_{1}, \ldots, \lambda_{d}}\left(X_{1}, \ldots, X_{d}\right)=\bigcap_{k=1}^{d} S_{\lambda_{k}}\left(X_{k}\right)$. Since $D^{\infty}\left(A_{1}, \ldots, A_{d}\right)=\bigcap_{k=1}^{d} D^{\infty}\left(A_{k}\right)$, the corollary follows.

Remark 15. Theorems 8,12 and Corollary 13 and their proofs are as well valid with anticommutators instead of commutators.

\section{Gevrey spaces, Lie algebras of operators and their ideals}

In this section we present several conditions such that equality (1) holds in case there are sufficiently many ideals. (cf. Corollary 9.) Let $d_{1}, d_{2} \in \mathbb{N}$ 
and let $X_{1}, \ldots, X_{d_{1}}, Y_{1}, \ldots, Y_{d_{2}}$ be skew-Hermitian operators in a Hilbert space defined on a common invariant domain. Suppose

$$
\left[X_{i}, Y_{j}\right] \in \operatorname{span}\left(\left\{Y_{1}, \ldots, Y_{d_{2}}\right\}\right)
$$

for all $i \in V_{1}:=\left\{1, \ldots, d_{1}\right\}$ and $j \in V_{2}:=\left\{1, \ldots, d_{2}\right\}$. In the proofs of intersection theorems of the form

$$
\begin{aligned}
& S_{\lambda_{1}, \ldots, \lambda_{d_{1}}, \mu_{1}, \ldots, \mu_{d_{2}}}\left(X_{1}, \ldots, X_{d_{1}}, Y_{1}, \ldots, Y_{d_{2}}\right) \\
& \quad=S_{\lambda_{1}, \ldots, \lambda_{d_{1}}}\left(X_{1}, \ldots, X_{d_{1}}\right) \cap S_{\mu_{1}, \ldots, \mu_{d_{2}}}\left(Y_{1}, \ldots, Y_{d_{2}}\right),
\end{aligned}
$$

we want to write $X_{\alpha} Y_{\beta}$ as a sum of terms of the form $c Y_{\delta} X_{\gamma}$. To this end, for $\alpha, \alpha^{\prime} \in M\left(V_{1}\right)$ define $\alpha^{\prime} \leq \alpha$ if $\alpha^{\prime}$ is obtained by deleting indices from the multi-index $\alpha$. Let $\alpha \backslash \alpha^{\prime}$ be the complementary part in $\alpha$. Moreover, for $\alpha=\left(j_{1}, \ldots, j_{n}\right) \in M\left(V_{1}\right)$ define $D^{\alpha}$ by

$$
D^{\alpha}:=\operatorname{ad} X_{j_{1}} \ldots \text { ad } X_{j_{n}} .
$$

The following lemma is due to Nelson ([11, Lemma 2.1]).

LemMA 16. Let $\alpha \in M\left(V_{1}\right)$ and $\beta \in M\left(V_{2}\right)$. Then

$$
X_{\alpha} Y_{\beta}=\sum_{\substack{\alpha^{\prime} \in M\left(V_{1}\right) \\ \alpha^{\prime} \leq \alpha}} D^{\alpha \backslash \alpha^{\prime}}\left(Y_{\beta}\right) X_{\alpha^{\prime}}
$$

If $\left[X_{i}, Y_{j}\right] \in \operatorname{span}\left(\left\{Y_{1}, \ldots, Y_{j}\right\}\right)$ for all $k, j$, we obtain that $D^{\alpha}\left(Y_{\beta}\right)$ is a sum of $Y_{\gamma}$. In order to determine which $\gamma$ occur, we introduce the concept of positive mutation. A positive mutation is a function $\tau: V_{2} \times V_{2} \rightarrow \mathbb{N}_{0}$ such that

$$
\tau(v, w)=0 \text { for all } v, w \in V_{2} \text { with } v<w .
$$

Let $\alpha, \beta \in M\left(V_{2}\right)$ and $k \in \mathbb{N}_{0}$. We say that $\alpha$ is connected with $\beta$ via a positive mutation of length $k$ if there exists a positive mutation $\tau$ on $V_{2}$ such that

$$
\left[\begin{array}{l}
\|\alpha\|_{v}+\sum_{w>v} \tau(w, v)-\sum_{w<v} \tau(v, w)=\|\beta\|_{v} \quad \text { for all } v \in V_{2} \\
\sum_{v, w \in V_{2}} \tau(v, w)=k
\end{array}\right.
$$

REMARK. If $\alpha$ is connected with $\beta$ via a positive mutation of length $k$, then in general it is not true that $\beta$ is connected with $\alpha$ via a positive mutation of length $k$. Also the positive mutation $\tau$ need not be unique and it is well possible that $\alpha$ is connected with $\beta$ via a positive mutation of length $l$ with $l \in \mathbb{N}_{0}, l \neq k$. 
Lemma 17. I. Let $\alpha, \beta \in M\left(V_{2}\right)$ and let $k \in \mathbb{N}_{0}$. Suppose $\alpha$ is connected with $\beta$ via a positive mutation of length $k$. Then $\|\alpha\|=\|\beta\|$.

II. Let $n \in \mathbb{N}, \alpha_{1}, \ldots, \alpha_{n}, \beta_{1}, \ldots, \beta_{n} \in M\left(V_{2}\right)$ and let $k_{1}, \ldots, k_{n} \in \mathbb{N}_{0}$. Suppose $\alpha_{i}$ is connected with $\beta_{i}$ via a positive mutation of length $k_{i}$ for all $i \in\{1, \ldots, n\}$. Then $\left\langle\alpha_{1}, \ldots, \alpha_{n}\right\rangle$ is connected with $\left\langle\beta_{1}, \ldots, \beta_{n}\right\rangle$ via a positive mutation of length $\sum_{i=1}^{n} k_{i}$.

III. Let $\alpha, \beta, \gamma \in M\left(V_{2}\right)$ and let $k, l \in \mathbb{N}_{0}$. Suppose $\alpha$ (respectively $\beta$ ) is connected with $\beta$ (respectively $\gamma$ ) via a positive mutation of length $k$ (respectively l). Then $\alpha$ is connected with $\gamma$ via a positive mutation of length $k+l$. (Transitivity.)

Proof. I: trivial, II: induction, III: trivial. (Take $\tau=\tau_{1}+\tau_{2}$.)

Lemma 18. I. There exist constants $M \geq 1$ and $c_{\beta, \gamma}^{\alpha} \in \mathbb{R}$, where $\alpha \in$ $M\left(V_{1}\right)$ and $\beta, \gamma \in M\left(V_{2}\right),\|\beta\|=\|\gamma\|$ such that

$\left|c_{\beta, \gamma}^{\alpha}\right| \leq(M\|\beta\|)^{\|\alpha\|}$ for all $\alpha \in M\left(V_{1}\right)$ and $\beta, \gamma \in M\left(V_{2}\right)$ with $\|\beta\|=\|\gamma\|$.

II. Suppose $\left[X_{i}, Y_{j}\right] \in \operatorname{span}\left(\left\{Y_{1}, \ldots, Y_{j}\right\}\right)$ for all $i \in\left\{1, \ldots, d_{2}\right\}$ and $j \in\left\{1, \ldots, d_{2}\right\}$. Then the constants $c_{\beta, \gamma}^{\alpha}$ in $I$ can be chosen such that in addition: for all $\alpha \in M\left(V_{1}\right)$ and $\beta, \gamma \in M\left(V_{2}\right)$ with $c_{\beta, \gamma}^{\alpha} \neq 0$ we have that $\beta$ is connected with $\gamma$ via a positive mutation of length $\|\alpha\|$.

Proof. There exist (possibly non-unique) constants $c_{i, j, k} \in \mathbb{R}$, where $i \in V_{1}$ and $j, k \in V_{2}$ such that

$$
\left[X_{i}, Y_{j}\right]=\sum_{k=1}^{d_{2}} c_{i, j, k} Y_{k}
$$

for all $i \in V_{1}$ and $j \in V_{2}$. Let $M_{0}:=1+\max \left\{\left|c_{i, j, k}\right|: i \in V_{1}, j, k \in V_{2}\right\}$. For all $\beta, \gamma \in M\left(V_{2}\right)$ take $c_{\beta, \gamma}^{()}:=1$ if $\beta=\gamma$ and $c_{\beta, \gamma}^{()}:=0$ if $\beta \neq \gamma$. Take $c_{(),()}^{\alpha}:=0$ for all $\alpha \in M\left(V_{1}\right) \backslash_{V_{1}^{0}}$. Then (6) and (7) hold for all $\alpha \in M\left(V_{1}\right)$ and $\beta, \gamma \in M\left(V_{2}\right)$ such that $\alpha=()$ or $\beta=()$ or $\gamma=()$.

Let $m \in \mathbb{N}$. Now we want to choose suitable constants $c_{\beta, \gamma}^{\alpha}$ such that (6) and (7) hold for all $\alpha \in M\left(V_{1}\right)$ with $\alpha \neq\left(\right.$ ) and $\beta, \gamma \in M\left(V_{2}\right)$ with $\|\beta\|=\|\gamma\|=m$. Let $i \in V_{1}, p \in\{1, \ldots, m\}$ and let $k \in V_{2}$. For $\delta=\left(j_{1}, \ldots, j_{m}\right) \in V_{2}^{m} \subset M\left(V_{2}\right)$ define

$$
f_{p, k}(\delta):=\left(j_{1}, \ldots, j_{p-1}, k, j_{p+1}, \ldots, j_{m}\right)
$$


and

$$
g_{i, p, k}(\delta):=c_{i, j_{p}, k} .
$$

Let $n \in \mathbb{N}, \alpha \in V_{1}^{n}$ and let $\beta \in V_{2}^{m}$. Let $\alpha=\left(i_{1}, \ldots, i_{n}\right)$. It follows by induction to $n$ that

$$
\begin{aligned}
D^{\alpha}\left(Y_{\beta}\right)= & \sum_{p_{1}=1}^{m} \sum_{k_{1}=1}^{d_{2}} \cdots \sum_{p_{n}=1}^{m} \sum_{k_{n}=1}^{d_{2}} g_{i_{n}, p_{n}, k_{n}}(\beta) \cdot g_{i_{n-1}, p_{n-1}, k_{n-1}}\left(f_{p_{n}, k_{n}}(\beta)\right) \cdots \\
& \cdots g_{i_{1}, p_{1}, k_{1}}\left(f_{p_{2}, k_{2}} \circ \cdots \circ f_{p_{n}, k_{n}}(\beta)\right) Y_{f_{p_{1}, k_{1}} \circ \cdots \circ f_{p_{n}, k_{n}}(\beta)} .
\end{aligned}
$$

Now the definitions of $c_{\beta, \gamma}^{\alpha}$, with $\gamma \in V_{2}^{m}$ speak for themselves. For $\gamma \in V_{2}^{m}$ we define

$$
\begin{aligned}
c_{\beta, \gamma}^{\alpha}:= & \sum g_{i_{n}, p_{n}, k_{n}}(\beta) \cdot g_{i_{n-1}, p_{n-1}, k_{n-1}}\left(f_{p_{n}, k_{n}}(\beta)\right) \cdots \\
& \cdots g_{i_{1}, p_{1}, k_{1}}\left(f_{p_{2}, k_{2}} \circ \cdots \circ f_{p_{n}, k_{n}}(\beta)\right)
\end{aligned}
$$

where the sum is over all $p_{1}, \ldots, p_{n} \in\{1, \ldots, m\}$ and $k_{1}, \ldots, k_{n} \in V_{2}$ such that $\gamma=f_{p_{1}, k_{1}} \circ \cdots \circ f_{p_{n}, k_{n}}(\beta)$. This proves (6), and clearly $\left|c_{\beta, \gamma}^{\alpha}\right| \leq$ $m^{n} d_{2}^{n} M_{0}^{n}=\left(d_{2} M_{0}\|\beta\|\right)^{\|\alpha\|}$.

II. Now suppose that $\left[X_{i}, X_{j}\right] \in\left(\left\{Y_{1}, \ldots, Y_{j}\right\}\right)$ for all $i \in V_{1}$ and $j \in V_{2}$. Then the constants in (8) can be chosen such that $c_{i, j, k}=0$ for all $j, k \in V_{2}$ with $k>j$. We define $c_{\beta, \gamma}^{\alpha}$ as in I. Let $\alpha \in M\left(V_{1}\right), \beta, \gamma \in M\left(V_{2}\right)$ and suppose $c_{\beta, \gamma}^{\alpha} \neq 0$. We may as well assume that $\alpha \neq(), \beta \neq()$ and $\gamma \neq()$. Let $m:=\|\beta\|=\|\gamma\|$ and let $i_{1}, \ldots, i_{n} \in V_{1}$ be such that $\alpha=\left(i_{1}, \ldots, i_{n}\right)$. Let $f_{p, k}$ and $g_{i, p, k}$ be as in $\mathbf{I}$.

Let $\delta=\left(j_{1}, \ldots, j_{m}\right) \in V_{2}^{m}$, let $i \in V_{1}, p \in\{1, \ldots, m\}, k \in V_{2}$ and suppose $g_{i, p, k}(\delta) \neq 0$. Then $c_{i, j_{p}, k} \neq 0$. This implies that $k \leq j_{p}$ and so $\delta=$ $\left(j_{1}, \ldots, j_{p}, \ldots, j_{m}\right)$ is connected with $f_{p, k}(\delta)=\left(j_{1}, \ldots, j_{p-1}, k, j_{p+1}\right.$, $\left.\ldots, j_{m}\right)$ via a positive mutation of length 1 . (Take as mutation $\tau(v, w):=1$ if $v=j_{p}$ and $w=k$ and $\tau(v, w):=0$ otherwise.)

Since $c_{\beta, \gamma}^{\alpha} \neq 0$ there exists $p_{1}, \ldots, p_{n} \in\{1, \ldots, m\}$ and $k_{1}, \ldots, k_{n} \in$ $V_{2}$ such that

$$
g_{i_{n}, p_{n}, k_{n}}(\beta) \cdot g_{i_{n-1}, p_{n-1}, k_{n-1}}\left(f_{p_{n}, k_{n}}(\beta)\right) \cdots g_{i_{1}, p_{1}, k_{1}}\left(f_{p_{2}, k_{2}} \circ \cdots \circ f_{p_{n}, k_{n}}(\beta)\right) \neq 0
$$

and

$$
\gamma=f_{p_{1}, k_{1}} \circ \cdots \circ f_{p_{n}, k_{n}}(\beta) .
$$

Then $g_{i_{n}, p_{n}, k_{n}}(\beta) \neq 0$ and $\ldots$ and $g_{i_{1}, p_{1}, k_{1}}\left(f_{p_{2}, k_{2}} \circ \cdots \circ f_{p_{n}, k_{n}}(\beta)\right) \neq 0$. Because $g_{i_{n}, p_{n}, k_{n}}(\beta) \neq 0$ we obtain that $\beta$ is connected with $f_{p_{n}, k_{n}}(\beta)$ via a positive mutation of length 1 . Because $g_{i_{n-1}, p_{n-1}, k_{n-1}}\left(f_{p_{n}, k_{n}}(\beta)\right) \neq 0$ 
we obtain that $f_{p_{n}, k_{n}}(\beta)$ is connected with $f_{p_{n-1}, k_{n-1}}\left(f_{p_{n}, k_{n}}(\beta)\right)$ via a positive mutation of length 1 . Then by Lemma 17.III, $\beta$ is connected with $f_{p_{n-1}, k_{n-1}}\left(f_{p_{n}, k_{n}}(\beta)\right.$ via a positive mutation of length 2 . By induction, $\beta$ is connected with $f_{p_{1}, k_{1}} \circ \cdots \circ f_{p_{n}, k_{n}}(\beta)=\gamma$ via a positive mutation of length $n=\|\alpha\|$.

Lemma 19. Let $\beta, \gamma \in M\left(V_{2}\right)$, let $j \in \mathbb{N}_{0}$ and let $0 \leq \mu_{1} \leq \mu_{2} \leq \cdots \leq$ $\mu_{d_{2}}$. Suppose $\beta$ is connected with $\gamma$ via a positive mutation of length $j$. Then

$$
\gamma !^{\mu} \leq\left(2^{d_{2} \mu_{d_{2}}}\right)^{\|\beta\|+j} \beta !^{\mu} .
$$

Proof. In this lemma we write $d:=d_{2}$. Let $\tau$ be a positive mutation on $V_{2}$ such that

$$
\|\beta\|_{v}+\sum_{w>v} \tau_{w, v}-\sum_{w<v} \tau_{v, w}=\|\gamma\|_{v} \quad \text { for all } v \in V_{2}
$$

and

$$
\sum_{v, w \in V_{2}} \tau_{v, w}=j
$$

where $\tau_{v, w}:=\tau(v, w)$, for all $v, w \in V_{2}$. Then by the inequalities $(n+m) ! \leq 2^{n+m} n ! m !$ and $(n-m) ! \leq n ! m !^{-1}$ we obtain

$$
\begin{aligned}
& y !^{\mu}=\left(\|\beta\|_{1}+\tau_{2,1}+\cdots+\tau_{d, 1}\right) !^{\mu_{1}} \cdot\left(\|\beta\|_{2}+\tau_{3,2}+\cdots+\tau_{d, 2}-\tau_{2,1}\right) !^{\mu_{2}} \cdot \\
& \cdots\left(\|\beta\|_{d}-\tau_{d, 1}-\cdots-\tau_{d, d-1}\right) !^{\mu_{d}} \\
& \leq\left(2^{\mu_{1}}\right)^{(d-1)\left(\|\beta\|_{1}+\tau_{2,1}+\cdots+\tau_{d, 1}\right)}\|\beta\|_{1} !^{\mu_{1}} \tau_{2,1} !^{\mu_{1}} \cdots \tau_{d, 1} !^{\mu_{1}} \cdot \\
& \cdot\left(2^{\mu_{1}}\right)^{(d-2)\left(\|\beta\|_{2}+\tau_{3,2}+\cdots+\tau_{d, 2}\right)}\|\beta\|_{2} !^{\mu_{2}} \tau_{3,2} !^{\mu_{2}} \cdots \tau_{d, 2} !^{\mu_{2}} \cdot \tau_{2,1} !^{-\mu_{2}} \\
& \cdots\|\beta\|_{d} !^{\mu_{d}} \tau_{d, 1} !^{-\mu_{d}} \cdots \tau_{d, d-1} !^{-\mu_{d}} \\
& \leq\left(2^{\mu_{d}}\right)^{d\left(\|\beta\|_{1}+\cdots+\|\beta\|_{d-1}+\sum_{v, w} \tau_{v, w}\right)} \prod_{v, w \in V_{2}} \frac{\tau_{v, w} !^{\tau_{w, w}} !^{\mu_{v}}}{v>w} \\
& \leq\left(2^{d \mu_{d}}\right)^{\|\beta\|+j} \beta !^{\mu} .
\end{aligned}
$$

Note that the ordering of the $\mu$ 's is essentially used in the last inequality only.

Lemma 20. Let $p \in \mathbb{N}$ and $k_{1}, \ldots, k_{p}, j_{1}, \ldots, j_{p} \in \mathbb{N}_{0}$. Let $j_{0}:=0$. Let $k:=k_{1}+\cdots+k_{p}$. Suppose $j_{i} \leq k_{i}+j_{i-1}$ for all $i \in\{1, \ldots, p\}$. Then

$$
\left(\begin{array}{c}
k_{1}+j_{0} \\
j_{1}
\end{array}\right) \cdots\left(\begin{array}{c}
k_{p}+j_{p-1} \\
j_{p}
\end{array}\right) \leq \frac{k !}{j_{p} !} \frac{1}{\left(k_{1}+j_{0}-j_{1}\right) !} \cdots \frac{1}{\left(k_{p}+j_{p-1}-j_{p}\right) !} .
$$


Proof.

$$
\begin{aligned}
& \left(\begin{array}{c}
k_{1}+j_{0} \\
j_{1}
\end{array}\right) \cdots\left(\begin{array}{c}
k_{p}+j_{p-1} \\
j_{p}
\end{array}\right) \\
& \quad=\frac{k_{1} ! \cdots k_{p} !}{\left(k_{1}-j_{1}\right) ! \cdots\left(k_{p}+j_{p-1}-j_{p}\right) !}\left(\begin{array}{c}
k_{2}+j_{1} \\
k_{2}
\end{array}\right) \cdots\left(\begin{array}{c}
k_{p}+j_{p-1} \\
k_{p}
\end{array}\right) \frac{1}{j_{p} !} \\
& \quad \leq \frac{k_{1} ! \cdots k_{p} !}{\left(k_{1}-j_{1}\right) ! \cdots\left(k_{p}+j_{p-1}-j_{p}\right) !}\left(\begin{array}{c}
k_{2}+k_{1} \\
k_{2}
\end{array}\right) \cdots\left(\begin{array}{c}
k_{p}+\cdots+k_{1} \\
k_{p}
\end{array}\right) \frac{1}{j_{p} !} \\
& \quad=\frac{k !}{j_{p} !} \frac{1}{\left(k_{1}+j_{0}-j_{1}\right) !} \cdots \frac{1}{\left(k_{p}+j_{p-1}-j_{p}\right) !} .
\end{aligned}
$$

This proves the lemma.

We now prove the main theorem of this section.

TheOREM 21. Let $d_{1}, d_{2} \in \mathbb{N}$ and let $X_{1}, \ldots, X_{d_{1}}, Y_{1}, \ldots, Y_{d_{2}}$ be skewHermitian operators in a Hilbert space defined on a common invariant domain. Suppose

$$
\left[X_{i}, Y_{j}\right] \in \operatorname{span}\left(\left\{Y_{1}, \ldots, Y_{d_{2}}\right\}\right)
$$

for all $i \in\left\{1, \ldots, d_{1}\right\}$ and $j \in\left\{1, \ldots, d_{2}\right\}$. Let $\lambda_{1}, \ldots, \lambda_{d_{1}} \geq 1$ and let $0 \leq \mu_{1} \leq \cdots \leq \mu_{d_{2}}$. Then

$$
\begin{aligned}
& S_{\lambda_{1}, \ldots, \lambda_{d_{1}}, \mu_{1}, \ldots, \mu_{d_{2}}}\left(X_{1}, \ldots, X_{d_{1}}, Y_{1}, \ldots, Y_{d_{2}}\right) \\
& \quad=S_{\lambda_{1}, \ldots, \lambda_{d_{1}}}\left(X_{1}, \ldots, X_{d_{1}}\right) \cap S_{\mu_{1}, \ldots, \mu_{d_{2}}}\left(Y_{1}, \ldots, Y_{d_{2}}\right)
\end{aligned}
$$

in the following two cases:

$$
\text { I. } \mu_{1}=\cdots=\mu_{d_{2}} \text {. }
$$

II. $\left[X_{i}, Y_{j}\right] \in \operatorname{span}\left(\left\{Y_{1}, \ldots, Y_{j}\right\}\right)$ for all $i \in\left\{1, \ldots, d_{1}\right\}$ and $j \in$ $\left\{1, \ldots, d_{2}\right\}$.

Note that $\mu_{j}$ may be taken smaller than 1 .

Proof. Let $V_{1}:=\left\{1, \ldots, d_{1}\right\}, V_{2}:=\left\{1, \ldots, d_{2}\right\}$ and let $M \geq 1$ and let $c_{\beta, \gamma}^{\alpha}$ be as in Lemma 18. Let $u \in S_{\lambda_{1}, \ldots, \lambda_{d_{1}}}\left(X_{1}, \ldots, X_{d_{1}}\right) \cap S_{\mu_{1}, \ldots, \mu_{d_{2}}}\left(Y_{1}, \ldots\right.$, $\left.Y_{d_{2}}\right)$. Then there exist $c, t>0$ such that

$$
\begin{array}{ll}
\left\|X_{\alpha} u\right\| \leq c t^{\|\alpha\|} \alpha !^{\lambda} & \text { for all } \alpha \in M\left(V_{1}\right), \\
\left\|Y_{\beta} u\right\| \leq c t^{\|\beta\|} \beta !^{\mu} & \text { for all } \delta \in M\left(V_{2}\right) .
\end{array}
$$

Let $p \in \mathbb{N}, \alpha_{1}, \ldots, \alpha_{p} \in M\left(V_{1}\right)$ and $\beta_{1}, \ldots, \beta_{p} \in M\left(V_{2}\right)$. Let $Z:=$ $X_{\alpha_{1}} Y_{\beta_{1}} \circ \cdots \circ X_{\alpha_{p}} Y_{\beta_{p}}$. We will consider $(Z u, u)$. By Lemmas 16 and 18 we 
obtain

$$
X_{\alpha} Y_{\beta}=\sum_{\substack{\alpha^{\prime} \in M\left(V_{1}\right) \\ \alpha^{\prime} \leq \alpha}} \sum_{\substack{\gamma \in M\left(V_{2}\right) \\\|\gamma\|=\|\beta\|}} c_{\beta, \gamma}^{\alpha \backslash \alpha^{\prime}} Y_{\gamma} X_{\alpha^{\prime}}
$$

for all $\alpha \in M\left(V_{1}\right)$ and $\beta \in M\left(V_{2}\right)$. So $Z$ is the sum of

$$
c_{\beta_{1}, \gamma_{1}}^{\delta_{1} \backslash \delta_{1}^{\prime}} \cdots c_{\beta_{p}, \gamma_{p}}^{\delta_{p} \backslash \delta_{p}^{\prime}} Y_{\left\langle\gamma_{1}, \ldots, \gamma_{p}\right\rangle} X_{\delta_{p}^{\prime}}
$$

where the sum is over all $\delta_{1}^{\prime} \in M\left(V_{1}\right)$ with $\delta_{1}^{\prime} \leq \delta_{1}$ and $\delta_{1}:=\alpha_{1}$, and all $\gamma_{1} \in M\left(V_{2}\right)$ with $\left\|\gamma_{1}\right\|=\left\|\beta_{1}\right\|$, over all $\delta_{2}^{\prime} \in M\left(V_{1}\right)$ with $\delta_{2}^{\prime} \leq \delta_{2}$ and $\delta_{2}:=\left\langle\delta_{1}^{\prime}, \alpha_{2}\right\rangle$, and all $\gamma_{2} \in M\left(V_{2}\right)$ with $\left\|\gamma_{2}\right\|=\left\|\beta_{2}\right\|, \ldots$, over all $\delta_{p}^{\prime} \in M\left(V_{1}\right)$ with $\delta_{p}^{\prime} \leq \delta_{p}$ and $\delta_{p}:=\left\langle\delta_{p-1}^{\prime}, \alpha_{p}\right\rangle$, and all $\gamma_{p} \in M\left(V_{2}\right)$ with $\left\|\gamma_{p}\right\|=\left\|\beta_{p}\right\|$. Consider one term of the sum

$$
c_{\beta_{1}, \gamma_{1}}^{\delta_{1} \backslash \delta_{1}^{\prime}} \cdots c_{\beta_{p}, \gamma_{p}}^{\delta_{p} \backslash \delta_{p}^{\prime}} Y_{\left\langle\gamma_{1}, \ldots, \gamma_{p}\right\rangle} X_{\delta_{p}^{\prime}}
$$

which is not zero and which corresponds to the tuple $\delta_{1}^{\prime}, \gamma_{1}, \ldots, \delta_{p}^{\prime}, \gamma_{p}$. Let $j_{i}:=\left\|\delta_{i}^{\prime}\right\|, k_{i}:=\left\|\alpha_{i}\right\|$ and $m_{i}:=\left\|\beta_{i}\right\|=\left\|\gamma_{i}\right\|$ for all $i \in\{1, \ldots, p\}$. Let $j_{0}:=0$. Let $k:=k_{1}+\cdots+k_{p}$ and $m:=m_{1}+\cdots+m_{p}$. Let $\alpha:=$ $\left\langle\alpha_{1}, \ldots, \alpha_{p}\right\rangle, \beta:=\left\langle\beta_{1}, \ldots, \beta_{p}\right\rangle$ and $\gamma:=\left\langle\gamma_{1}, \ldots, \gamma_{p}\right\rangle$. Then $j_{i} \leq\left\|\delta_{i}\right\|=$ $k_{i}+j_{i-1}$, for all $i \in\{1, \ldots, p\}$. Moreover, $k=\|\alpha\|$ and $m=\|\beta\|=\|\gamma\|$. By Schwarz' inequality we obtain that

$$
\begin{aligned}
\left|\left(Y_{\left\langle y_{1}, \ldots, \gamma_{p}\right)} X_{\delta_{p}^{\prime}} u, u\right)\right| & =\left|\left(X_{\delta_{p}^{\prime}} u, Y_{\gamma^{r}} u\right)\right| \leq\left\|X_{\delta_{p}^{\prime}} u\right\|\left\|Y_{\gamma^{r}} u\right\| \\
& \leq c^{2} t^{\left\|\delta_{p}^{\prime}\right\|+m} \delta_{p}^{\prime} !^{\lambda} \cdot \gamma !^{\mu} \leq c^{2} t^{k+m} \delta_{p}^{\prime} !^{\lambda} \cdot \gamma !^{\mu} .
\end{aligned}
$$

Let $l(v):=\|\alpha\|_{v}-\left\|\delta_{p}^{\prime}\right\|_{v}$ for all $v \in V_{1}$. Then $l(v) \geq 0$ and $\sum_{v \in V_{1}} l(v)=$ $k-j_{p}$. So

$$
\begin{aligned}
\delta_{p}^{\prime} !^{\lambda} & \leq \alpha !^{\lambda}(l(1)) !^{-\lambda_{1}} \cdots\left(l\left(d_{1}\right)\right) !^{-\lambda_{d_{1}}} \\
& \leq \alpha !^{\lambda}(l(1)) !^{-1} \cdots\left(l\left(d_{1}\right)\right) !^{-1} \\
& \leq \alpha !^{\lambda} 2^{d_{1}\left(k-j_{p}\right)}\left(k-j_{p}\right) !^{-1}
\end{aligned}
$$

This is the only place where we use that $\lambda_{1}, \ldots, \lambda_{d_{1}} \geq 1$.

Next we estimate the factor $\gamma !^{\mu}$ in the cases I and II.

Case I. Suppose $\mu_{1}=\cdots=\mu_{d_{2}}$. Then $\gamma !^{\mu} \leq\|\gamma\| !^{\mu_{d_{2}}}=\|\beta\| !^{\mu_{d_{2}}} \leq$ $2^{\mu_{d_{2}} d_{2} m} \beta !^{\mu}$.

Case II. Suppose $\left[X_{i}, Y_{j}\right] \in \operatorname{span}\left(\left\{Y_{1}, \ldots, Y_{j}\right\}\right)$ and all $i \in V_{1}$ and $j \in$ $V_{2}$. This case needs more care. Now we can use Lemma 18 .II and so we may assume that the constants $c_{\beta, \gamma}^{\alpha}$ are as in Lemma 18.II. Recall that we 
are considering a non-zero term of a large sum. So the coefficients $c_{\boldsymbol{\beta}_{i}, \gamma_{i}}^{\delta_{i} \backslash \delta_{i}^{\prime}}$ are not zero for all $i \in\{1, \ldots, p\}$. Hence by Lemma 18.II we obtain that $\beta_{i}$ is connected with $\gamma_{i}$ via a positive mutation of length $\left\|\delta_{i} \backslash \delta_{i}^{\prime}\right\|=$ $k_{i}+j_{i-1}-j_{i}$. Therefore by Lemma 17.II, $\beta=\left\langle\beta_{1}, \ldots, \beta_{p}\right\rangle$ is connected with $\gamma=\left\langle\gamma_{1}, \ldots, \gamma_{p}\right\rangle$ via a positive mutation of length $k-j_{p}$. But then by Lemma 19,

$$
\gamma !^{\mu} \leq\left(2^{d_{2} \mu_{d_{2}}}\right)^{m+k-j_{p}} \beta !^{\mu} \leq\left(2^{d_{2} \mu_{d_{2}}}\right)^{m+k} \beta !^{\mu} .
$$

Having estimated $\gamma !^{\mu}$ in the two cases we obtain that $\gamma !^{\mu} \leq\left(2^{d_{2} \mu_{d_{2}}}\right)^{k+m} \beta !^{\mu}$. Then

$$
\left|\left(Y_{\left\langle\gamma_{1}, \ldots, \gamma_{p}\right\rangle} X_{\delta_{p}^{\prime}} u, u\right)\right| \leq c^{2}\left(b_{1} t\right)^{k+m}\left(k-j_{p}\right) !^{-1} \alpha !^{\lambda} \cdot \beta !^{\mu},
$$

where $b_{1}:=2^{d_{1}+d_{2} \mu_{d_{2}}}$.

We count the number of terms in the sum. There are $\left(\begin{array}{c}k_{i}+j_{i-1} \\ j_{i}\end{array}\right)$ multiindices $\delta_{i}^{\prime}$ with $\delta_{i}^{\prime} \leq \delta_{i}$ and $\left\|\delta_{i}^{\prime}\right\|=j_{i}$ for all $i \in\{1, \ldots, p\}$. There are $d_{2}^{m_{i}}$ multi-indices $\gamma_{i} \in M\left(V_{2}\right)$ with $\left\|\gamma_{i}\right\|=\left\|\beta_{i}\right\|=m_{i}$. Furthermore, $\left|c_{\beta_{i}}^{\delta_{i} \backslash \delta_{i}^{\prime}}\right| \leq\left(M m_{i}\right)^{\left\|\delta_{i} \backslash \delta_{i}^{\prime}\right\|}=\left(M m_{i}\right)^{k_{i}+j_{i-1}-j_{i}}$ for all $i \in\{1, \ldots, p\}$. Hence we obtain by the triangle inequality and Lemma 20 :

$$
\begin{aligned}
|(Z u, u)| \leq & \sum_{j_{1}=0}^{k_{1}+j_{0}} \cdots \sum_{j_{p}=0}^{k_{p}+j_{p-1}}\left(\begin{array}{c}
k_{1}+j_{0} \\
j_{1}
\end{array}\right) \cdots\left(\begin{array}{c}
k_{p}+j_{p-1} \\
j_{p}
\end{array}\right) d_{2}^{m_{1}+\cdots+m_{p}} \\
& \cdot\left(M m_{1}\right)^{k_{1}+j_{0}-j_{1}} \cdots\left(M m_{p}\right)^{k_{p}+j_{p-1}-j_{p}} c^{2}\left(b_{1} t\right)^{k+m}\left(k-j_{p}\right) !^{-1} \alpha !^{\lambda} \cdot \beta !^{\mu} \\
& \leq c^{2}\left(b_{1} d_{2} M t\right)^{k+m} \alpha !^{\lambda} \cdot \beta !^{\mu} \\
& \cdot \sum_{j_{1}=0}^{k_{1}+j_{0}} \cdots \sum_{j_{p}=0}^{k_{p}+j_{p-1}} \frac{k !}{j_{p} !\left(k-j_{p}\right) !} \frac{m_{1}^{k_{1}+j_{0}-j_{1}} \cdots m_{p}^{k_{p}+j_{p-1}-j_{p}}}{\left(k_{1}+j_{0}-j_{1}\right) ! \cdots\left(k_{p}+j_{p-1}-j_{p}\right) !} \\
& \leq c^{2}\left(2 e b_{1} d_{2} M t\right)^{k+m} \alpha !^{\lambda} \cdot \beta !^{\mu}
\end{aligned}
$$

Since $\left\|\left\langle\alpha_{1}, \ldots, \alpha_{p}\right\rangle\right\|+\left\|\left\langle\beta_{1}, \ldots, \beta_{p}\right\rangle\right\|=k+m$, this proves the theorem by Lemma 1.

COROLLARY 22. Let $\mathfrak{g}$ be a real Lie algebra of skew-hermitian operators in a Hilbert space defined on a common invariant domain. Let $d_{1}, d_{2} \in \mathbb{N}$ and let $X_{1}, \ldots, X_{d_{1}}, Y_{1}, \ldots, Y_{d_{2}} \in \mathfrak{g}$. Suppose $\mathfrak{g}=\operatorname{span}\left(\left\{X_{1}, \ldots, X_{d_{1}}, Y_{1}, \ldots\right.\right.$, $\left.\left.Y_{d_{2}}\right\}\right)$ and suppose that $\operatorname{span}\left(\left\{Y_{1}, \ldots, Y_{d_{2}}\right\}\right)$ is an ideal in $\mathfrak{g}$. Let $\lambda_{1}, \ldots, \lambda_{d_{1}}$ $\geq 1$ and let $\mu \geq 0$. Then

$$
\begin{aligned}
& S_{\lambda_{1}, \ldots, \lambda_{d_{1}}, \mu, \ldots, \mu}\left(X_{1}, \ldots, X_{d_{1}}, Y_{1}, \ldots, Y_{d_{2}}\right) \\
& \quad=S_{\lambda_{1}, \ldots, \lambda_{d_{1}}}\left(X_{1}, \ldots, X_{d_{1}}\right) \cap S_{\mu, \ldots, \mu}\left(Y_{1}, \ldots, Y_{d_{2}}\right) .
\end{aligned}
$$


Let $\mathfrak{g}$ be a Lie algebra and let $X_{1}, \ldots, X_{d}$ be a basis in $\mathfrak{g}$. The basis $X_{1}, \ldots, X_{d}$ is called an ordered basis in $\mathfrak{g}$ if

$$
\left[X_{i}, X_{j}\right] \in \operatorname{span}\left(\left\{X_{1}, \ldots, X_{\min (i, j)}\right\}\right)
$$

for all $i, j \in\{1, \ldots, d\}$. Not every solvable Lie algebra has an ordered basis; but every Lie algebra which has an ordered basis is solvable. Every nilpotent Lie algebra has an ordered basis. (For example, any Jordan-Hölder basis.)

COROllary 23. Let $\mathfrak{g}$ be a real Lie algebra of skew-Hermitian operators in a Hilbert space defined on a common invariant domain. Let $X_{1}, \ldots, X_{d}$ be an ordered basis in $g$ and let $\lambda_{d} \geq 1$ and $\lambda_{d-1} \geq \cdots \geq \lambda_{2} \geq \max \left(\lambda_{1}, 1\right) \geq$ $\lambda_{1} \geq 0$. Then

$$
S_{\lambda_{1}, \ldots, \lambda_{d}}\left(X_{1}, \ldots, X_{d}\right)=\bigcap_{k=1}^{d} S_{\lambda_{k}}\left(X_{k}\right) \text {. }
$$

\section{Non-triviality of certain Gevrey spaces}

In this section we present several Gevrey spaces which are dense in the corresponding Banach space. Let $\pi$ be a representation of a Lie group $G$ in a Banach space $E$. For each $X$ in the Lie algebra $\mathfrak{g}$ of $G$ let $d \pi(X)$ denote the infinitesimal generator of the one parameter group $t \mapsto \pi_{\exp t X}$. We emphasize that for $X, Y \in \mathfrak{g}$ the operators $d \pi(X)$ and $d \pi(Y)$ need not have the same domain. In this connection there is the following theorem.

THEOREM 24. Let $\pi$ be a representation of a Lie group $G$ in a Banach space $E$. Let $X_{1}, \ldots, X_{d}$ be a basis in the Lie algebra $\mathfrak{g}$ of $G$. Then $S_{1}, \ldots,{ }_{1}\left(d \pi\left(X_{1}\right), \ldots, d \pi\left(X_{d}\right)\right)$ is dense in $E$. Moreover, for all $\lambda \geq 1$ the space $S_{\lambda, \ldots, \lambda}\left(d \pi\left(X_{1}\right), \ldots, d \pi\left(X_{d}\right)\right)$ consists of all $u \in E$ such that the map $x \mapsto \pi_{x} u$ is a Gevrey function of order $\lambda$ from $G$ into $E$.

Proof. See [11, Theorem 4] and [10, Proposition 1.5].

Now suppose the Lie group $G$ is nilpotent. A basis $X_{1}, \ldots, X_{d}$ in $\mathfrak{g}$ is called a Jordan-Hölder basis in $\mathfrak{g}$ if

$$
\left[X_{i}, X_{j}\right] \in \operatorname{span}\left(\left\{X_{1}, \ldots, X_{\min (i, j)-1}\right\}\right)
$$

for all $i, j \leq d$. Corollary 23 has the following form for unitary representations. 
THEOREM 25. Let $\pi$ be a unitary representation of a nilpotent Lie group $G$. Let $X_{1}, \ldots, X_{d}$ be a Jordan-Hölder basis in $\mathfrak{g}$. Let $\lambda_{d} \geq 1$ and $\lambda_{d-1} \geq$ $\cdots \geq \lambda_{2} \geq \max \left(\lambda_{1}, 1\right) \geq \lambda_{1} \geq 0$. Then $S_{\lambda_{1}, \ldots, \lambda_{d}}\left(d \pi\left(X_{1}\right), \ldots, d \pi\left(X_{d}\right)\right)=$ $\bigcap_{k=1}^{d} S_{\lambda_{k}}\left(d \pi\left(X_{k}\right)\right)$.

Proof. Since $D^{\infty}\left(d \pi\left(X_{1}\right), \ldots, d \pi\left(X_{d}\right)\right)=\bigcap_{k=1}^{d} D^{\infty}\left(d \pi\left(X_{k}\right)\right)$ by [7, Theorem 1.1], the theorem follows immediately from Corollary 23 .

The Gevrey spaces considered in the above theorem are dense in the Hilbert space if $\lambda_{1} \geq 1$ according to Theorem 24 . In this section we prove that these spaces are dense in the Hilbert space if $\lambda_{2}, \ldots, \lambda_{d} \geq 1$ and $\lambda_{1}>0$.

In case the representation $\pi$ is irreducible, we can even take $\lambda_{1}=0$. Indeed, because $X_{1}$ belongs to the center of $\mathfrak{g}$ and $d \pi\left(X_{1}\right)$ is closed, it follows by Taylor, [14, Chapter 0 Propositions 4.3 and 4.5] that there exists $\alpha \in \mathbb{C}$ such that $d \pi\left(X_{1}\right)=\alpha I$. So $S_{0,1, \ldots, 1}\left(d \pi\left(X_{1}\right), \ldots, d \pi\left(X_{d}\right)\right)=$ $S_{1,1, \ldots, 1}\left(d \pi\left(X_{1}\right), \ldots, d \pi\left(X_{d}\right)\right)$ which is dense. In general, for nonirreducible representations $\pi$, the operator $d \pi\left(X_{1}\right)$ is not bounded.

Now let $X_{1}, \ldots, X_{d}$ be a fixed Jordan-Hölder basis in $\mathfrak{g}$ and let $\pi$ be a (not necessarily irreducible) unitary representation of $G$ in a Hilbert space $H$. Let $\mathfrak{g}_{c}$ be the complexification of $\mathfrak{g}$ and let $G_{c}$ be a connected simply connected complex Lie group with Lie algebra $\mathfrak{g}_{c}$. (See [15, Theorem 3.15.1].) Let exp denote the exponential map from $\mathfrak{g}_{c}$ onto $G_{c}$. Without loss of generality we may assume that $G=\exp (\mathfrak{g})$. For all $k \in\{1, \ldots, d\}$ define $g_{k}: \mathbb{C} \rightarrow G_{c}$ by

$$
g_{k}(z):=\exp \left(z X_{k}\right) \quad(z \in \mathbb{C}) .
$$

Define $g: \mathbb{C}^{d} \rightarrow G_{c}$ by

$$
g\left(z_{1}, \ldots, z_{d}\right):=g_{1}\left(z_{1}\right) \cdots g_{d}\left(z_{d}\right) \quad\left(z_{1}, \ldots, z_{d} \in \mathbb{C}\right) .
$$

By [15, Theorem 3.18.11], the map $g$ is an analytic diffeomorphism from $\mathbb{C}^{d}$ onto $G_{c}$ and the map $\left.g\right|_{\mathbb{R}^{d}}$ is an analytic diffeomorphism from $\mathbb{R}^{d}$ into $G$.

Lemma 26. Let $k \in\{1, \ldots, d\}$. Then there exists polynomials $P_{k, j}: \mathbb{C}^{k-j}$ $\rightarrow \mathbb{C}$, where $j \in\{1, \ldots, k-2\}$, such that for all $z \in \mathbb{C}$ and all $t_{1}, \ldots, t_{d} \in \mathbb{C}$ we have

$$
g_{k}(z) g\left(t_{1}, \ldots, t_{d}\right)=g\left(s_{1}, \ldots, s_{d}\right)
$$


with

$$
\begin{aligned}
s_{j} & =t_{j}+P_{k, j}\left(z, t_{j+1}, \ldots, t_{k-1}\right) & & \text { if } j<k-1, \\
s_{k-1} & =t_{k-1}, & & \\
s_{k} & =t_{k}+z, & & \\
s_{j} & =t_{j} & & \text { if } j>k .
\end{aligned}
$$

Proof. See [8, Lemma 5.1].

TheOREM 27. Let $G$ be a nilpotent Lie group with Lie algebra $\mathfrak{g}$. Let $\pi$ be a unitary representation of $G$ in a Hilbert space $H$ and let $X_{1}, \ldots, X_{d}$ be a Jordan-Hölder basis in $\mathfrak{g}$. Let $\lambda \in(0,1)$. Then $S_{\lambda, 1, \ldots, 1}\left(d \pi\left(X_{1}\right), \ldots\right.$, $\left.d \pi\left(X_{d}\right)\right)$ is a dense subspace of $H$.

Proof. We may suppose that $G$ is connected and simply connected. Let $g_{c}, G_{c}, g_{k}$ and $g$ be as above. Let the polynomials $P_{k, j}$ be as in Lemma 26. Let $m$ be the maximum of the degrees of these polynomials $P_{k, j}$. Let $p_{0}=p_{-1}=0$ and for $k \in\{1, \ldots, d\}$ let $p_{k}:=(1+m)^{k-1}(1-\lambda)^{-1}$, let $q_{k}:=\max \left(p_{k}, m p_{k-2}\right)$, and let $\lambda_{k}:=1-q_{k}^{-1}$. Observe that $\lambda_{1}=\lambda$. Then $q_{k} \geq p_{k} \geq(1-\lambda)^{-1}>1$ for all $k \in\{2, \ldots, d\}$, hence $\lambda_{k}>0$. We shall prove that $\bigcap_{k=1}^{d} S_{\lambda_{k}}\left(d \pi\left(X_{k}\right)\right)$ is dense in $H$. Since all $\lambda_{k}<1$, then also $S_{\lambda_{1}}\left(d \pi\left(X_{1}\right)\right) \cap \bigcap_{k=2}^{d} S_{1}\left(d \pi\left(X_{k}\right)\right)$ is dense in $H$. Hence by Theorems 24 and $25, S_{\lambda, 1, \ldots, 1}\left(d \pi\left(X_{1}\right), \ldots, d \pi\left(X_{d}\right)\right)=S_{\lambda_{1}}\left(d \pi\left(X_{1}\right)\right) \cap \bigcap_{k=2}^{d} S_{1}\left(d \pi\left(X_{k}\right)\right)$ is dense in $H$.

Consider the following assertion.

There exists a dense set $Z_{2}$ of $L^{1}(G)$ such that

$$
\pi(f)(H) \subset \bigcap_{k=1}^{d} S_{\lambda_{k}}\left(d \pi\left(X_{k}\right)\right) \quad \text { for all } f \in Z_{2} .
$$

Because the representation $\pi$ is continuous, $\bigcup_{f \in Z_{2}} \pi(f)(H)$ is dense in $H$ and having proved this assertion, the proof of the theorem is completed.

Let $Z$ be the set of all entire functions $F$ on $\mathbb{C}^{d}$ for which there exist constants $A, B, C>0$ (depending on $F$ ) such that

$$
\left|F\left(z_{1}, \ldots, z_{d}\right)\right| \leq C \exp \left[-A \sum_{k=1}^{d}\left|\operatorname{Re} z_{k}\right|^{p_{k}}+B \sum_{k=1}^{d}\left|\operatorname{Im} z_{k}\right|^{p_{k}}\right]
$$

for all $\left(z_{1}, \ldots, z_{d}\right) \in \mathbb{C}^{d}$. Since $p_{1}, \ldots, p_{d}>1$, the set $\left\{\left.F\right|_{\mathbf{R}^{d}}: F \in Z\right\}$ is dense in $L^{1}\left(\mathbb{R}^{d}\right)$ according to [5, Section IV.9]. Let $Z_{1}:=\left\{f \in \mathbb{C}^{G_{c}}: f \circ g \in\right.$ 
$Z\}$ and $Z_{2}:=\left\{\left.f\right|_{G}: f \in Z_{1}\right\}$. By [13, page 90], the map $f \mapsto \int_{\mathbf{R}^{d}} f(g(z)) d z$ is a Haar integral on $G$, hence the set $Z_{2}$ is dense in $L^{1}(G)$. Similarly, $Z_{1}$ is dense in $L^{1}\left(G_{c}\right)$ and in particular, a subset of $L^{1}\left(G_{c}\right)$.

Let $f \in Z_{1}$, let $F:=f \circ g$ and let $A, B, C$ be constants corresponding to $F$. Let $k \in\{1, \ldots, d\}$. There exists $C_{1}>0$ such that for all $j \in$ $\{1, \ldots, d\}$ with $j<k-1$, all $t_{1}, \ldots, t_{d} \in \mathbb{R}$ and all $z \in \mathbb{C}$ we have

$$
\begin{gathered}
\left|\operatorname{Im} s_{j}\right| \leq C_{1}\left(1+\sum_{l=j+1}^{k-1}\left|t_{l}\right|^{m}+|z|^{m}\right), \\
\left|\operatorname{Re} s_{j}\right| \geq\left|t_{j}\right|-C_{1}\left(1+\sum_{l=j+1}^{k-1}\left|t_{l}\right|^{m}+|z|^{m}\right)
\end{gathered}
$$

with $s_{j}:=t_{j}+P_{k, j}\left(z, t_{j+1}, \ldots, t_{k-1}\right)$. Using the inequalities $|a+b|^{p} \leq$ $2^{p}\left(|a|^{p}+|b|^{p}\right)$ and $-|a-b|^{p} \leq-2^{-p}|a|^{p}+|b|^{p}$ for all $a, b \in \mathbb{R}$ and $p \geq 1$, and using the fact that $1 \leq p_{1} \leq \cdots \leq p_{d}$, we obtain by Lemma 26 and a straightforward calculation that for all $t_{1}, \ldots, t_{d} \in \mathbb{R}$ and all $z \in \mathbb{C}$,

$\left|f\left(g_{k}(z) g\left(t_{1}, \ldots, t_{d}\right)\right)\right| \leq C_{2} \exp \left[-A_{1} \sum_{j=1}^{d}\left|t_{j}\right|^{p_{j}}+B_{1} \sum_{j=1}^{d}\left|t_{j}\right|^{m p_{j-1}}+C_{3}|z|^{q_{k}}\right]$,

where $A_{1}, B_{1}, C_{2}, C_{3}$ are positive constants which depend only on $A, B, C$, $C_{1}$ and $p_{1}, \ldots, p_{d}$. For $z \in \mathbb{C}$ define $T_{z} f: G \rightarrow \mathbb{C}$ by

$$
\left(T_{z} f\right)(x):=f\left(g_{k}(z) x\right) \quad(x \in G) .
$$

Since $m p_{j-1}<p_{j}$ for all $j \in\{1, \ldots, d\}$, we have $T_{z} f \in L^{1}(G)$ for all $z \in$ $\mathbb{C}$. Hence the map $t \mapsto T_{t} f$ from $\mathbb{R}$ into $L^{1}(G)$ extends holomorphically to an entire function from $\mathbb{C}$ into $L^{1}(G)$ of exponential order $\leq q_{k}$. So the map $t \mapsto L_{\exp \left(t X_{k}\right)}\left(\left.f\right|_{G}\right)=T_{-t} f$ from $\mathbb{R}$ into $L^{1}(G)$ extends holomorphically to an entire function from $\mathbb{C}$ into $L^{1}(G)$ of exponential order $\leq q_{k}=$ $\left(1-\lambda_{k}\right)^{-1}$. Then by [9, Corollary 4.1], $\pi\left(\left.f\right|_{G}\right) u \in S_{\lambda_{k}}\left(d \pi\left(X_{k}\right)\right)$ for all $u \in H$. This proves the assertion.

COROLLARY 28. Let $\pi$ be a unitary representation of a nilpotent Lie group $G$ in a Hilbert space $H$. Let $X_{1}, \ldots, X_{d}$ be a Jordan-Hölder basis in the Lie algebra of $G$. Let $\lambda_{2}, \ldots, \lambda_{d} \geq 1$ and let $\lambda_{1}>0$. Then $S_{\lambda_{1}, \ldots, \lambda_{d}}\left(d \pi\left(X_{1}\right)\right.$, $\left.\ldots, d \pi\left(X_{d}\right)\right)$ is dense in $H$. 


\section{Example: the $a x+b$ group}

The $a x+b$ group is the topological space $G:=(0, \infty) \times \mathbb{R}$ endowed with the multiplication $(a, b) \cdot(c, d):=(a c, a d+b),(a, b),(c, d) \in G$. So $G$ is a Lie group which is isomorphic with the group of matrices of the form $\left(\begin{array}{ll}a & b \\ 0 & 1\end{array}\right)$ with $a>0$ and $b \in \mathbb{R}$. Let $y$ denote the embedding from $G$ into $\mathbf{R}^{2}$. By $e:=(1,0)$ we denote the identity in $G$. Define

$$
X:=\left.\frac{\partial}{\partial y_{1}}\right|_{e}, \quad Y:=\left.\frac{\partial}{\partial y_{2}}\right|_{e} .
$$

Then $X, Y$ is a basis in the Lie algebra $\mathfrak{g}$ of $G$ and $[X, Y]=Y$. So by Theorem 21.II and [7, Theorem 1.1] we obtain for every unitary representation $\pi$ in a Hilbert space, all $\lambda \geq 1$ and all $\mu \geq 0$ that

$$
S_{\lambda, \mu}(d \pi(X), d \pi(Y))=S_{\lambda}(d \pi(X)) \cap S_{\mu}(d \pi(Y)) .
$$

The $a x+b$ group has up to unitary equivalence two irreducible infinite dimensional representations. For $(a, b) \in G$ there exists a unique unitary map $U_{(a, b)}^{ \pm}$from $L^{2}(\mathbb{R})$ onto $L^{2}(\mathbb{R})$ such that

$$
\left[U_{(a, b)}^{ \pm} f\right](x)=e^{ \pm i b e^{x}} f(x+\log a) \text { a.e. } x \in \mathbb{R}
$$

for all $f \in C_{c}(\mathbb{R})$. Then $(a, b) \mapsto U_{(a, b)}^{ \pm}$is a (continuous unitary) irreducible representation of $G$ in $L^{2}(\mathbb{R})$. Let $D$ be the skew-adjoint differentiation operator in $L^{2}(\mathbb{R})$. Let $E$ be the multiplication operator in $L^{2}(\mathbb{R})$ of multiplication by the function $x \mapsto e^{x}, x \in \mathbb{R}$. Then $d U^{ \pm}(X)=D$ and $d U^{ \pm}(Y)= \pm i E$.

For $\lambda, \mu \geq 0$ we shall consider the Gevrey space $S_{\lambda, \mu}\left(d U^{ \pm}(X), d U^{ \pm}(Y)\right)$ $=S_{\lambda, \mu}(D, E)$. Since $S_{\lambda, \mu}(D, E) \subset D^{\infty}(D, E) \subset D^{\infty}(D)$, every element of $S_{\lambda, \mu}(D, E)$ is infinitely differentiable.

Lemma 29. Let $\lambda, \mu \geq 0$. Let $f \in S_{\lambda, \mu}(D, E)$. Then there exist $C, t>0$ such that for all $k, l \in \mathbb{N}_{0}$ :

$$
\left\|E^{k} D^{l} f\right\|_{\infty} \leq C t^{k+l} k !^{\mu} l !^{\lambda} .
$$

Proof. By a classical Sobolev inequality we obtain for all $k, l \in \mathbb{N}_{0}$ :

$$
\begin{aligned}
\left\|E^{k} D^{l} f\right\|_{\infty} & \leq \sqrt[4]{\pi / 2}\left\|E^{k} D^{l} f\right\|_{2}+\sqrt[4]{\pi / 2}\left\|D E^{k} D^{l} f\right\|_{2} \\
& \leq \sqrt[4]{\pi / 2} c(1+t)\left(2^{\lambda} t\right)^{k+l} k !^{\mu} l !^{\lambda},
\end{aligned}
$$

where $c, t>0$ are some constants. 
Paley and Wiener ([12, Theorems I and IV]) have given a characterization of the space $S_{1}(D)$ in terms of analytic functions. Because of our intersection theorems, the characterizations of the spaces $S_{1}(D)$ as derived by Paley and Wiener is useful in the characterization of the spaces $S_{1, \mu}(D, E)$ where $\mu>0$.

THEOREM 30. Let $f \in L^{2}(\mathbb{R})$. Then $f \in S_{1}(D)$ is and only if there exist $t>0$ such that $f$ can be extended to an analytic function $F$ on the strip $\{z \in \mathbb{C}:|\operatorname{Im} z|<2 t\}$ and

$$
\sup _{y \in(-t, t)} \int_{-\infty}^{\infty}|F(x+i y)|^{2} d x<\infty
$$

THEOREM 31. Let $\mu>0$ and $f \in L^{2}(\mathbb{R})$. The following conditions are equivalent:

I. $f \in S_{1, \mu}(D, E)$,

II. $f \in S_{1}(D)$ and there exist $C, \sigma, t>0$ such that $f$ can be extended to an analytic function $F$ on the strip $\{z \in \mathbb{C}:|\operatorname{Im} z|<2 t\}$ and

$$
|F(x+i y)| \leq C e^{-\sigma e^{x / \mu}}
$$

for all $x \in \mathbb{R}$ and all $y \in(-t, t)$.

Proof. I $\Rightarrow$ II. This can be proved in the same manner as in [5, Section IV.2] a characterization has been given for the space $S_{\alpha}^{1}$.

II $\Rightarrow$ I. Let $k \in \mathbb{N}$. Then

$$
\begin{aligned}
\int_{\mathbf{R}}\left|\left(e^{\frac{x}{\mu}}\right)^{k} f(x)\right|^{2} d x & \leq \sqrt{2 \pi}\|f\|_{2}^{2}+C^{2} \int_{0}^{\infty} e^{2 x k / \mu} e^{-2 \sigma e^{x / \mu}} d x \\
& \leq \sqrt{2 \pi}\|f\|_{2}^{2}+\mu C^{2} \sigma^{-2 k} k !^{2}<\infty .
\end{aligned}
$$

So $f \in S_{1}\left(|E|^{1 / \mu}\right)=S_{\mu}(E)$. (See Example 5.) Hence, by assumption, $f \in$ $S_{1}(D) \cap S_{\mu}(E)$. Now we use the intersection theorem in order to conclude that $f \in S_{1, \mu}(D, E)$.

Corollary 32. Let $\mu>0$. Define $W$ from $L^{2}(\mathbb{R})$ onto $L^{2}(\mathbb{R})$ by

$$
[W f](x):=\frac{1}{\sqrt{\mu}} f\left(\frac{x}{\mu}\right) \text { a.e. } x \in \mathbb{R}
$$

for all $f \in L^{2}(\mathbb{R})$. Then $W$ is a unitary map in $L^{2}(\mathbb{R})$ which maps $S_{1,1}(D, E)$ onto $S_{1, \mu}(D, E)$.

Corollary 33. Let $\mu>0$. Then the Gevrey space $S_{1, \mu}(D, E)$ is dense in $L^{2}(\mathbb{R})$. 
LEMMA 34. The space $S_{1,0}(D, E)$ is trivial.

Proof. Let $f \in S_{1,0}(D, E)$. By Lemma 29 there exist $C, t>0$ such that $\left\|E^{k} D^{l} f\right\|_{\infty} \leq C t^{k+l} l$ ! for all $k, l \in \mathbb{N}_{0}$. So $f$ can be extended to an analytic function $F$ on the strip $\left\{z \in \mathbb{C}:|\operatorname{Im} z|<t^{-1}\right\}$. Let $x \in \mathbb{R}$ be such that $e^{x}>t$. Then for all $k \in \mathbb{N}_{0}: e^{k x}|f(x)| \leq C t^{k}$, so $|F(x)|=|f(x)| \leq$ $\inf \left\{C\left(t e^{-x}\right)^{k}: k \in \mathbb{N}_{0}\right\}=0$. Hence $F=0$ and $f=0$.

Lemma 35. Let $\lambda \in[0,1)$ and $\mu>0$. Then the space $S_{\lambda, \mu}(D, E)$ is trivial.

Proof. Let $f \in S_{\lambda, \mu}(D, E)$. By Lemma 29 there exist $C, t>0$ such that $\left\|E^{k} D^{l} f\right\|_{\infty} \leq C t^{k+l} k !^{\mu} l !^{\lambda}$ for all $k, l \in \mathbb{N}_{0}$. As in [5, Section IV.2.2], it follows that $f$ can be extended to an entire function $F$ for which there exist $\tau, C_{1}>0$ such that

$$
|F(x+i y)| \leq C_{1} e^{-\sigma e^{x / \mu}+\tau|y|^{\frac{1}{1-\lambda}}}
$$

for all $x, y \in \mathbb{R}$. Let $z=x+i y \in \mathbb{C}$. Then

$|F(z) \cdot F(i z) \cdot F(-z) \cdot F(-i z)| \leq C_{1}^{4} e^{-\sigma e^{x / \mu}-\sigma e^{-x / \mu}+2 \tau|x|^{\frac{1}{1-\lambda}}} e^{-\sigma e^{y / \mu}-\sigma e^{-y / \mu}+2 \tau|y|^{\frac{1}{1-\lambda}}}$.

Hence $\lim _{|z| \rightarrow \infty} F(z) \cdot F(i z) \cdot F(-z) \cdot F(-i z)=0$. By the Liouville theorem, $F(z) \cdot F(i z) \cdot F(-z) \cdot F(-i z)=0$ for all $z \in \mathbb{C}$. Then also $F=0$ and $f=0$.

At this point it is not known whether the spaces $S_{\lambda, 0}(D, E)$ are trivial if $\lambda>1$. This problem will be solved in the following lemma.

LEMMA 36. Let $\lambda>1$. Then the space $S_{\lambda, 0}(D, E)$ is dense in $L^{2}(\mathbb{R})$. In particular, the Gelfand-Shilov space $S_{0}^{\lambda}$ is a subspace of $S_{\lambda, 0}(D, E)$.

Proof. Let $f \in S_{0}^{\lambda}$. Then there exist $t>0$ such that $f(x)=0$ for all $x \in \mathbb{R},|x|>t$. Therefore, for all $k \in \mathbb{N}_{0}$ :

$$
\int_{\mathbf{R}}\left|\left(e^{x}\right)^{k} f(x)\right|^{2} d x \leq\left(e^{t}\right)^{2 k} \sqrt{2 \pi}\|f\|_{2}^{2},
$$

hence $f \in S_{0}(E)$. Obviously, $f \in S_{0}^{\lambda} \subset S_{\lambda}(D)$. Therefore $f \in S_{\lambda}(D) \cap$ $S_{0}(E)$. Using again the intersection theorem, we obtain that $f \in S_{\lambda, 0}(D, E)$.

We summarize the results of the previous lemmas and corollary. 
THEOREM 37. Let $\lambda, \mu \geq 0$. Then the space $S_{\lambda, \mu}(D, E)$ is dense in $L^{2}(\mathbb{R})$ if and only if

$$
\lambda>1 \text { and } \mu \geq 0 \quad \text { or } \quad \lambda=1 \text { and } \mu>0 .
$$

\section{References}

[1] F. E. Browder, 'Real analytic functions on product spaces and separate analyticity', Canadian J. Math 13 (1961), 650-656.

[2] S. J. L. van Eijndhoven, 'Functional analytic characterizations of the Gelfand-Shilov spaces $S_{\alpha}^{\beta}$, , Proc. Kon. Nederl. Akad. Wetensch. A 90 (1987), 133-144.

[3] W. G. Faris, Self-adjoint operators, Lecture Notes in Math. 433 (Springer, Berlin, 1975).

[4] M. Flato, and J. Simon, 'Separate and joint analyticity in Lie groups representations', J. Funct. Anal. 13 (1973), 268-276.

[5] I. M. Gelfand, and G. E. Shilov, Generalized functions, vol. 2 (Academic Press, New York, 1968).

[6] M. Gevrey, 'Sur la nature analytique des solutions des équations aux dérivées partielles', Ann. Sci. École Norm. Sup. 35 (1918), 129-190.

[7] R. Goodman, 'Analytic and entire vectors for representations of Lie groups', Trans. Amer. Math. Soc. 143 (1969), 55-76.

[8] R. Goodman, 'Complex Fourier analysis on a nilpotent Lie group', Trans. Amer. Math. Soc. 160 (1971), 373-391.

[9] R. Goodman, 'Elliptic and subelliptic estimates for operators in an enveloping algebra', Duke Math. J. 47 (1980), 819-833.

[10] R. Goodman, and N. R. Wallach, 'Whittaker vectors and conical vectors', J. Funct. Anal. 39 (1980), 199-279.

[11] E. Nelson, 'Analytic vectors', Ann. of Math. 70 (1959), 572-615.

[12] R. E. A. C. Paley, and N. Wiener, Fourier transforms in the complex domain, Colloquium Publications 19 (Amer. Math. Soc., New York, 1934).

[13] L. Pukanszky, Leçons sur les représentations des groupes (Dunod, Paris, 1967).

[14] M. E. Taylor, Noncommutative harmonic analysis, Math. Surveys Monographs 22 (Amer. Math. Soc., Providence, 1986).

[15] V. S. Varadarajan, Lie groups, Lie algebras, and their representations, Graduate Texts in Math. 102 (Springer, New York, 1984).

[16] J. Wloka, Grundräume and verallgemeinerte Funktionen, Lecture Notes in Math. 82 (Springer, Berlin, 1969).

\section{Department of Mathematics}

Eindhoven University of Technology

P.O. Box 513

$5600 \mathrm{MB}$ Eindhoven

The Netherlands 